\title{
Small Forbidden Configurations III
}

\author{
R. P. Anstee* and N. Kamoosi ${ }^{\dagger}$ \\ Mathematics Department \\ The University of British Columbia \\ Vancouver, B.C. Canada V6T 1 Z2 \\ anstee@math.ubc.ca
}

Submitted: Nov 24, 2005; Accepted: Nov 7, 2007; Published: Nov 12, 2007

Mathematics Subject Classification: 05D05

\begin{abstract}
The present paper continues the work begun by Anstee, Ferguson, Griggs and Sali on small forbidden configurations. We define a matrix to be simple if it is a $(0,1)$-matrix with no repeated columns. Let $F$ be a $k \times l(0,1)$-matrix (the forbidden configuration). Assume $A$ is an $m \times n$ simple matrix which has no submatrix which is a row and column permutation of $F$. We define $\operatorname{forb}(m, F)$ as the largest $n$, which would depend on $m$ and $F$, so that such an $A$ exists. 'Small' refers to the size of $k$ and in this paper $k=2$. For $p \leq q$, we set $F_{p q}$ to be the $2 \times(p+q)$ matrix with $p\left[\begin{array}{l}1 \\ 0\end{array}\right]$ 's and $q\left[\begin{array}{l}0 \\ 1\end{array}\right]$ 's. We give new exact values: forb $\left(m, F_{0,4}\right)=\left\lfloor\frac{5 m}{2}\right\rfloor+2$, $\operatorname{forb}\left(m, F_{1,4}\right)=\left\lfloor\frac{11 m}{4}\right\rfloor+1$, forb $\left(m, F_{1,5}\right)=\left\lfloor\frac{15 m}{4}\right\rfloor+1$, forb $\left(m, F_{2,4}\right)=\left\lfloor\frac{10 m}{3}-\frac{4}{3}\right\rfloor$ and forb $\left(m, F_{2,5}\right)=4 m$ (For forb $\left(m, F_{1,4}\right)$, forb $\left(m, F_{1,5}\right)$ we obtain equality only for certain classes modulo 4$)$. In addition we provide a surprising construction which $\operatorname{shows}$ forb $\left(m, F_{p q}\right) \geq\left(\frac{p+q}{2}+O(1)\right) m$.
\end{abstract}

Keywords: forbidden configurations, extremal set theory, $(0,1)$-matrices.

\section{Introduction}

We define a simple matrix as a $(0,1)$-matrix with no repeated columns (such a matrix can be viewed as the incidence matrix of a set system). We say $A$ has a configuration $F$ if there is a submatrix of $A$ which is a row and column permutation of $F$. Our problems are of the following type: given a matrix $F$ and an $m \times n$ simple matrix $A$ which has

\footnotetext{
${ }^{*}$ Research supported in part by NSERC

${ }^{\dagger}$ Research supported by NSERC USRA; currently at Microsoft.
} 
no configuration $F$, determine an upper bound on $n$ which would depend on $m, F$. We denote the best possible upper bound as forb $(m, F)$. Alternatively, forb $(m, F)$ is the smallest function so that if $A$ is any simple $m \times(\operatorname{forb}(m, F)+1)$ matrix, then $A$ must have the configuration $F$.

Definition 1.1 Let $K_{k}$ denote the $k \times 2^{k}$ simple matrix of all columns on $k$ rows and let $K_{k}^{r}$ denote the $k \times\left(\begin{array}{l}k \\ r\end{array}\right)$ simple matrix of all columns with $r$ 1's.

Thus $K_{k}^{1}$ is a copy of the identity matrix (after row and column permutations) and $K_{k}^{0}$ is a column of $k 0$ 's. Also $K_{k}^{r}$ can be viewed as the complete $r$-uniform hypergraph on $k$ vertices. The problem of Forbidden Configurations is usually focused on $F=K_{k}$ and the problem of VC-dimension. A matrix $A$ has $V C$-dimension $k$ if it has a configuration $K_{k}$ and does not have a configuration $K_{k+1}$. A number of applications of VC-dimension exist including to Geometry (e.g.[6]) and to computational learning theory (e.g.[5]). Another related problem area is the investigation of forbidden patterns in $(0,1)$-matrices. The problem here is to determine the maximum number of 1's in a matrix given that the 1's do not form a certain pattern. A pattern can be given by a $(0,1)$-matrix and we say a matrix $A$ has a pattern $P$ if there is a submatrix $B$ of $A$ of the same size as $P$ that satisfies $B \geq P$. A number of problems are solved by Füredi and Hajnal in [4] and recently Tardos [7] has made much further progress here. These problems unfortunately appear have little direct connection with the problem of forbidden configurations. Configurations correspond to induced submatrices where patterns correspond to non-induced submatrices.

This paper considers particular choices of configurations $F$ namely $F_{p q}$ defined, for $p \leq q$, as

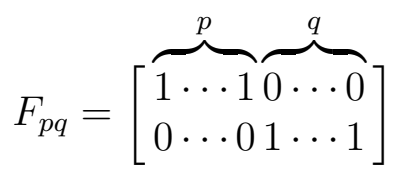

These are deceptively simple cases that demonstrate the rich structure associated with forbidden configurations. This paper establishes exact bounds for $F_{0,4}$ in Theorem 2.1, $F_{1,4}$ in Theorem 2.4, $F_{1,5}$ in Theorem 2.7, $F_{2,4}$ in Theorem 3.14, $F_{2,5}$ in Theorem 3.9 as well as a new asymptotic construction for $F_{p q}$ in Theorem 4.1, all listed in Table 1. Exact bounds are the Holy Grail of Extremal Set Theory. The arguments are rather intricate, making interesting use of graph theory. Our results establish that extremal matrices have much of their structure forced which is not typical for many forbidden configuration results. In [1] Anstee, Griggs and Sali established that forb $\left(m, F_{p q}\right)$ was linear in $m$ with constants depending on $p, q$. In [3] Anstee, Ferguson and Sali established a number of exact bounds.

Definition 1.2 Let $M_{m}$ denote an $m \times\left\lfloor\frac{m}{2}\right\rfloor$ simple matrix of columns each of two 1 's each where no row has more than one 1.

Such a matrix can be viewed as a matching on the rows.

Definition 1.3 Given two matrices $A, B$ we use the notation $A-B$ to denote the matrix obtained from $A$ by deleting columns that are also in $B$. 
This is equivalent to a set difference.

Definition 1.4 Let \#0's(A), \#1's(A) to denote the number of 0 's and the number of 1 's in $A$ respectively.

We are trying to build a set of tools that would yield exact or asymptotic results for all $F$, not just $F_{p q}$. It is interesting that the constructions for the exact bounds emphasize different characteristics than that of the asymptotically excellent general construction. From the point of view of proofs, transitivity (Lemma 1.7) is used heavily in the exact bounds but is not known to follow for general $F_{p q}$. Our current state of knowledge of forb $\left(m, F_{p q}\right)$ is summarized in Table 1, which contains results from [1],[3] as well as results from this paper.

Definition 1.5 Assume $A$ is a simple matrix with no $F_{p q}$. Let $R_{i}$ denote the ith row of $A$. We construct a graphlike structure $D(A)$ considering rows as vertices and having directed edges $i \rightarrow j$ if the number of $\left[\begin{array}{l}0 \\ 1\end{array}\right]$ 's in $\left[\begin{array}{l}R_{i} \\ R_{j}\end{array}\right] \leq p-1$. We have dotted edges $i \cdots j$ if the numbers of $\left[\begin{array}{l}0 \\ 1\end{array}\right]$ 's and $\left[\begin{array}{l}1 \\ 0\end{array}\right]$ 's in $\left[\begin{array}{l}R_{i} \\ R_{j}\end{array}\right]$ are each chosen from $\{p, p+1, \ldots, q-1\}$.

We follow the proof techniques of Theorem 2.8 in [3]. A more careful analysis of the components formed by the 'dotted' edges is required but much of the same structure is demonstrated. The proof of Theorem 3.9 fills in a few gaps in the original proof of Theorem 2.8, Claim iv) for $F_{2,3}$ in [3] where some extra comments would have made things clearer.

We begin with a series of Lemmas used in our inductive arguments.

Lemma 1.6 Deletion Lemma. Assume we are trying to show that forb $\left(m, F_{p q}\right) \leq$ $\mathrm{cm}+c^{\prime}$. We may assume that we cannot delete $k$ rows $(k<m)$ and up to ck columns and still have the resulting matrix $A_{m-k}$ be simple.

Proof: Assume $A$ is an $m \times n$ simple matrix and $A_{m-k}$ is an $(m-k) \times n^{\prime}$ simple matrix. Then by induction:

$$
n \leq n^{\prime}+c k \leq c(m-k)+c^{\prime}+c k=c m+c^{\prime} .
$$




\section{Table 1}

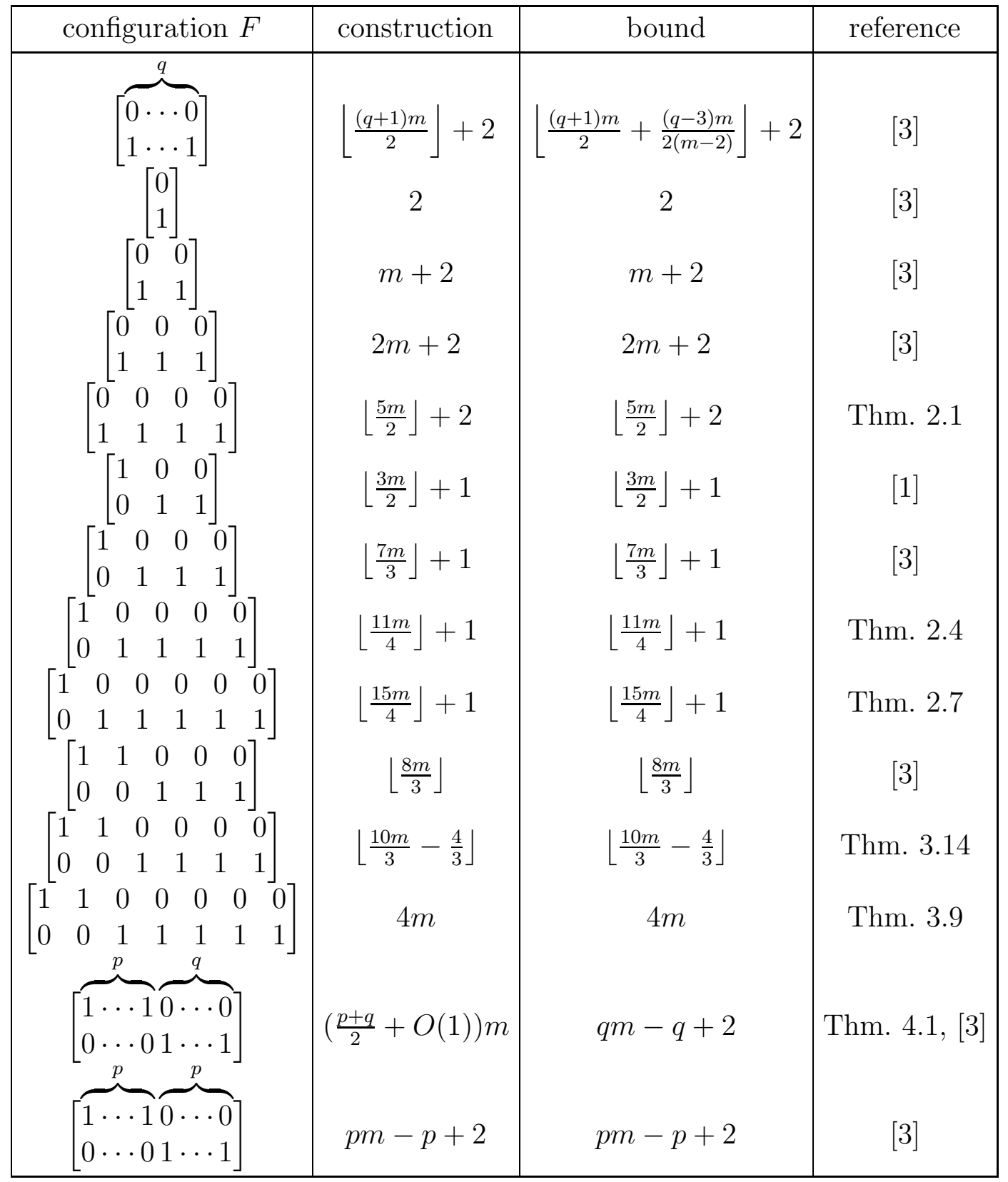

Lemma 1.7 Transitivity Lemma. If we are trying to show forb $\left(m, F_{p q}\right) \leq c m+c^{\prime}$, we may assume that for an $m$-rowed matrix $A$ with no configuration $F_{p q}$ then $D(A)$ has (i) For $c \geq 2(p-1)$, each pair of rows is connected by exactly one edge of $D(A)$. (ii) For $c \geq(2(p-1)+(q-1)) / 2$, the graph on the directed edges of $D(A)$ is transitive and contains no cycles.

Proof: For part (i), it is clear that each pair $i, j$ is joined by some edge: $i \rightarrow j, i \cdots j$, or $j \rightarrow i$. Our definition of $i \cdots j$ ensures that we do not have $i \rightarrow j$ or $j \rightarrow i$. If $i \rightarrow j$ and 
$j \rightarrow i$ then we can delete row $i$ and the up to $2(p-1)$ columns non-constant on rows $i, j$ and obtain a simple $(m-1)$-rowed matrix. Thus we are done by the Deletion Lemma 1.6 for

$$
2(p-1) \leq c,
$$

which was the assumption.

For part (ii), to show the graph on the directed edges is transitive and contains no cycles consider the case: $i \rightarrow j$ and $j \rightarrow k$. We have the three possibilities:
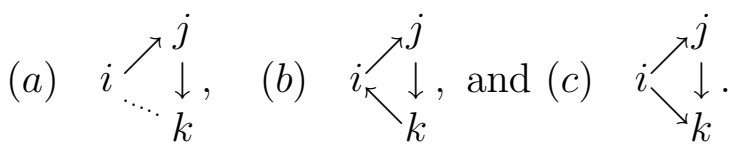

For cases (a) and (b) we look at the possible entries for these three rows. The entries above the braces indicate the number of possible columns of these types.

$$
\begin{aligned}
& i \overbrace{0 \cdots 00 \cdots 00}^{\leq p-1} \overbrace{0 \cdots 1 \cdots 1}^{\leq p-1} \overbrace{1 \cdots 11 \cdots 1}^{\leq q-1} 0 \cdots 01 \cdots 1 \\
& \text { j } 1 \cdots 11 \cdots 10 \cdots 00 \cdots 00 \cdots 01 \cdots 10 \cdots 01 \cdots 1 \text {. } \\
& k 0 \cdots 01 \cdots 11 \cdots 11 \cdots 10 \cdots 00 \cdots 00 \cdots 01 \cdots 1
\end{aligned}
$$

(in case (b), $\leq q-1$ would have been $\leq p-1)$. We can eliminate the two rows $i$ and $j$ and the at most $2(p-1)+(q-1)$ columns non-constant on rows $i, j, k$ to produce a simple matrix $A_{m-2}$. But then for

$$
2(p-1)+(q-1) \leq 2 c
$$

which was the assumption, we are done by the Deletion Lemma 1.6. Thus we may assume $A$ can have (c) only. It is straightforward to deduce that the graph induced by the directed edges must therefore be transitive and have no directed cycles.

Lemma 1.8 Ordering Lemma. If we are trying to show forb $\left(m, F_{p q}\right) \leq c m+c^{\prime}$ and the directed edges of $D(A)$ form a transitive graph with no pair $x \rightarrow y$ and $y \rightarrow x$, then the components formed by the dotted edges considered as an undirected graph, can be linearly ordered so that if the components are $C_{1}, C_{2}, C_{3}, \ldots$, then for any $i<j$ and any vertices $x \in C_{i}$ and $y \in C_{j}$ there is a directed edge $x \rightarrow y$.

Proof: We can verify this by showing that it is impossible to have directed edges $u \rightarrow v$, $v \rightarrow w$ with $u, w \in C_{i}$ and $v \in C_{j}$. We deduce $u \neq w$ by hypothesis and then we can assume there is a shortest path of dotted edges (in $C_{i}$ ) joining $u, w$. But then for some adjacent pair of vertices $x, y \in C_{i}$ with $x \cdots \cdots$ and yet $x \rightarrow v, v \rightarrow y$. This contradicts transitivity.

With respect to the components and the ordering, the following definitions are given for canonical with respect to $C_{i}$ and hence non-canonical columns. Also t-varied columns are defined which for $t \geq 1$ are called varied columns and for $t=0$ are called flat columns. 
Definition 1.9 Assume that the rows of $A$ have been ordered to respect the ordering from the Ordering Lemma 1.8. We say that a column of $A$ is canonical with respect to a component $C_{i}$ if it has all 1's on components $C_{j}$ with $j<i$ and all 0 's on components $C_{j}$ with $j>i$ (i.e. all 1's above and all 0's below). Any other column which is non-constant on $C_{i}$ is non-canonical.

The following definition is first used in Section 3.

Definition 1.10 For a column $\alpha$ of $A$, define $n(\alpha)=t$ if there are exactly $t$ components $C_{i_{1}}, C_{i_{2}}, \ldots, C_{i_{t}}$ with the column non-constant on $C_{i_{j}}$ for each $j$ with $1 \leq j \leq t$. We say $\alpha$ is t-varied when $n(\alpha)=t$. Define a column to be flat if it is 0-varied and define a column to be varied if it is t-varied for $t \geq 1$.

The following bound (4) is equation (2) in the Appendix of [3].

Lemma 1.11 Upper Bound Lemma. Let $B$ be a $k \times n(0,1)$-matrix with no column of all 1's and no column of all O's. Assume $B$ has no pair of rows which differ in more than $t$ columns i.e. $B$ has at most $t$ disjoint configurations $F_{0,1}$ on the same pair of rows. Then

$$
n \leq \frac{t k}{2} \text {. }
$$

If $B$ is simple and $t \geq 4$ then

$$
n \leq\left\lfloor 2 k+\frac{(t-4) k(k-1)}{4(k-2)}\right\rfloor .
$$

Proof: Each column contributes at least $k-1$ configurations $F_{0,1}$ in the $k$ rows. More than $t k / 2$ of such columns would give more than $t k(k-1) / 2$ of the $F_{0,1}$ configurations in $k(k-1) / 2$ pairs of rows in $B$. One pair of rows would then contain more than $t$ configurations, a contradiction yielding (3).

If $B$ is simple then we note there are at most $2 k$ columns which have only $k-1$ configurations, namely the columns with at most one 1 and the columns with at most one 0 . All other columns have at least $2(k-2)$ configurations $F_{0,1}$. We deduce $2 k(k-1)+$ $(n-2 k) 2(k-2) \leq t k(k-1) / 2$ and so we obtain $(4)$.

Note that if we wish to forbid, for example, the configuration $F_{0,5}$ in a simple $k \times n$ matrix $B$, then we can use $t=8$ in (4) in the Upper Bound Lemma 1.11.

\section{Exact Bounds for $F_{0,4}, F_{1,4}$ and $F_{1,5}$}

To handle $F_{1,4}, F_{2,4}$ we need the (unsurprising) bound for $F_{0,4}$ that requires some care.

Theorem 2.1 For $F_{0,4}=\left[\begin{array}{llll}0 & 0 & 0 & 0 \\ 1 & 1 & 1 & 1\end{array}\right]$,

$$
\operatorname{forb}\left(m, F_{0,4}\right)=\left\lfloor\frac{5 m}{2}\right\rfloor+2 \text { for } m \geq 4 \text {. }
$$

THE ELECTRONiC JoURnal of COMBINATORICs 14 (2007), \#R79 
Proof: To prove the lower bound forb $\left(m, F_{0,4}\right) \geq\left\lfloor\frac{5 m}{2}\right\rfloor+2$ we use the construction $\left[K_{m}^{0} K_{m}^{1} M_{m} K_{m}^{m-1} K_{m}^{m}\right]$ for $m \geq 4$.

To prove the upper bound forb $\left(m, F_{0,4}\right) \leq\left\lfloor\frac{5 m}{2}\right\rfloor+2$, we appeal to the Upper Bound Lemma 1.11 with $t=6$ to obtain forb $\left(m, F_{0,4}\right) \leq 2+2 m+\frac{m}{2}+\frac{m}{2(m-2)}$ which yields equality for $m$ even and $m \geq 6$. To improve this bound by 1 in the other cases we have a general argument for $m \geq 6$ as well as specific arguments for $m=4,5$.

Assume $m$ is odd and $m \geq 7$. Let $A$ be an $m \times\left(2 m+\left\lfloor\frac{m+1}{2}\right\rfloor+2\right)$ matrix with no configuration $F_{0,4}$. We wish to arrive at a contradiction. Let $a_{i}$ denote the number of columns with either $i$ 's or $i$ 's for $i=1,2$ and let $a_{3}$ be the number of remaining columns. Following the Upper Bound Lemma 1.11,

$$
6\left(\begin{array}{c}
m \\
2
\end{array}\right)=3 m(m-1) \geq(m-1) a_{1}+2(m-2) a_{2}+3(m-3) a_{3},
$$

where $a_{1} \leq 2 m$.

If $a_{1}=2 m-1$, then $a_{2}+a_{3} \leq \frac{m+1}{2}+\frac{m+1}{2(m-2)}$ (noting that $2(m-2) \leq 3(m-3)$ for $m \geq 5)$. Thus for $m \geq 7$, we have $a_{1}+a_{2}+a_{3} \leq\left\lfloor\frac{5 m}{2}\right\rfloor+2$, a contradiction as desired. Values of $a_{1}<2 m-1$ can be ignored since $m-1<2 m-4<3 m-9$ for $m \geq 7$.

If $a_{1}=2 m$ we compute $3 m(m-1)=2 m(m-1)+\frac{m+1}{2} 2(m-2)+2$. Thus with $3(m-3)-2(m-2)>2$ for $m \geq 9$, we deduce that either $a_{2}=\frac{m+1}{2}$ and $a_{3}=0$ (this must be the case for $m \geq 9$ ) or possibly $m=7$ and $a_{2}=3$ and $a_{3}=1$. Assume that we are in such a case. Note that $A=\left[K_{m}^{0} K_{m}^{1} B K_{m}^{m-1} K_{m}^{m}\right]$ where $B$ is an $m \times \frac{m+1}{2}$ matrix consisting of columns of at least two 1's and two 0's. We deduce that $B$ has no configuration $F_{0,2}$ otherwise $A$ would have $F_{0,4}$. We may assume without loss of generality that $A$ has the first column with two 1's in the top 2 rows and 0's below (may need to reorder and to complement $A$ for this but we have $a_{2}>0$ ). To avoid creating a configuration $F_{0,2}$, we must have all remaining columns have 0's in the top 2 rows. Thus we could delete the top two rows from $B$ and the first column and get a new matrix $B^{\prime}$ with the same properties. By an inductive argument we can assume $B^{\prime}$ has at most $\left\lfloor\frac{(m-2)-1}{2}\right\rfloor$ columns and then $B$ has at most $\left\lfloor\frac{m-1}{2}\right\rfloor$ columns, a contradiction.

To verify equality for $m=4,5$ requires finite checking. We can follow the above argument for $m=4$ when $a_{1}=8$ and for $m=5$ when $a_{1}=10$. For $m=4$, we can verify $a_{1}=8$. For $m=5$, we may also have $a_{1}=9$ and $a_{2}=4$ and so assume this is so. Consider a new graph formed only by the columns with two 1's (think of the rows as vertices and the columns as edges). We can find a copy of $F_{0,4}$ and hence a contradiction if two edges are incident on a row $r$ unless the column of one 1 on row $r$ is not present in $A$. We can assume there are at least two edges (by complementing $A$ if necessary). Thus either we have two (not three) edges incident on row $r$ and up to one additional edge disjoint from these two edges (which means the column of one 1 on row $r$ is not present in $A$ ) or precisely two disjoint edges. In the former case there is only no way to add a column of three 1's. In the latter case a column with three 1's must have exactly one 1 in the pair of rows of each edge and a 1 in the remaining row. But now there is no way to have two such columns with three 1's. Either case yields a contradiction.

We now have verified the bound for all cases. 
We now present new exact bounds for $F_{1,4}, F_{1,5}$ which focus on somewhat different issues. Obtaining the exact bound for all $m$ eludes us for $F_{1,5}$.

Lemma 2.2 Let $m$ be given. For $m \not \equiv 3(\bmod 4)$ and $m \geq 4$ we have

$$
\operatorname{forb}\left(m, F_{1,4}\right) \geq\left\lfloor\frac{11 m}{4}\right\rfloor+1 \text {. }
$$

For $m \equiv 3(\bmod 4)$, we have

$$
\operatorname{forb}\left(m, F_{1,4}\right) \geq\left\lfloor\frac{11 m}{4}\right\rfloor
$$

Proof: We provide a construction for $A_{m}$, a simple $m \times\left\lfloor\frac{11 m}{4}\right\rfloor+1$ matrix which avoids the configuration $F_{1,4}$ for $m \geq 4$ and $m \not \equiv 3(\bmod 4)$. The following construction creates a simple matrix $A$ with no $F_{1,4}$ under the assumption that the smaller matrices have no $F_{1,4}$ so that the number of columns in $A$ is the sum of the number of columns of $A_{a}$ and $A_{b}$ minus 1. Assume $a+b=m$.

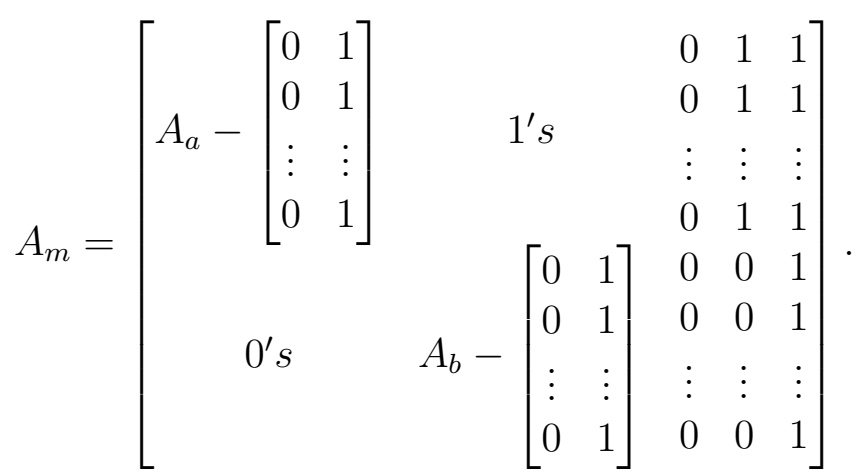

We can construct $A_{4}, A_{5}, A_{6}$ as $A_{k}=\left[K_{k}^{0} K_{k}^{1} M_{k} K_{k}^{k-1} K_{k}^{k}\right]$ (see Definitions 1.1, 1.2). $A_{m}$ can be constructed using (5) with $b \in\{4,5,6\}$ and $a=m-b \equiv 0(\bmod 4)$. For $m \equiv 3(\bmod 4)$, we choose $A_{3}=K_{3}$ and we can take $b=3$ and $a=m-b \equiv 0(\bmod 4)$.

Hence, we have forb $\left(m, F_{1,4}\right) \geq\left\lfloor\frac{11 m}{4}\right\rfloor+1$ for $m \geq 4$ and $m \not \equiv 3(\bmod 4)$ and forb $\left(m, F_{1,4}\right) \geq\left\lfloor\frac{11 m}{4}\right\rfloor$ for $m \equiv 3(\bmod 4)$.

We show forb $\left(m, F_{1,4}\right) \leq \frac{11 m}{4}+1$ by induction. It is easily verified for $m=1,2,3,4$. Assume $m \geq 5$ and proceed by induction. Let $A$ be a simple $m \times n$ matrix with no configuration $F_{1,4}$. We wish to show $n \leq \frac{11 m}{4}+1$.

We construct the structure $D(A)$ of Definition 1.5.

Lemma 2.3 Our structure $D(A)$ can be assumed to have the following properties.

(i) Each pair of rows is connected by exactly one edge, directed or dotted.

(ii) The graph on the directed edges is transitive and contains no cycles.

(iii) All components of the graph on the dotted edges are cliques of size at least 4.

Proof: In view of our bound $\frac{11 m}{4}+1$ i.e. $c=11 / 4$ with $p=1, q=4$, we have (i) and (ii) by the Transitivity Lemma 1.7 . 
As before, the components induced by the dotted edges can be ordered by the Ordering Lemma 1.8. Reorder the rows of $A$ (relabelling vertices of $D(A)$ ) to respect that order. With $p=1$, we have that for two rows $x, y$ with $x \in C_{i}$ and $y \in C_{j}$ and $i<j$ (in the ordering), then $x \rightarrow y$ and so there is no submatrix ${ }_{y}^{x}\left[\begin{array}{l}0 \\ 1\end{array}\right]$ as indicated on rows $x, y$. We note that if there is a column non-constant on a component $C_{i}$, then such a column is forced to be canonical with respect to $C_{i}$ (see Definition 1.9). Thus if we consider a component $C_{i}$ on $k$ vertices and let $A^{\prime \prime}$ be the submatrix of $A$ given by the $k$ rows of $C_{i}$ there is no repeated non-constant column. Thus a column of $A$ is either 1-varied or is flat (a flat column can have different values on different components) with 1's above 0's. For a given component $C_{i}$ on $k$ vertices, consider the matrix $A^{\prime}$ formed from $A$ by deleting the $k$ rows corresponding to $C_{i}$. If two columns of $A^{\prime}$ are identical then they arise from two columns of $A$ for which either: one of the columns is non-constant on $C_{i}$ or the two columns are both flat and have all 1's on components above $C_{i}$ and all 0's on components below and one column is all 0's on $C_{i}$ and one is all 1's on $C_{i}$. Thus if we have a component $C_{i}$ on $k$ vertices with $h$ columns non-constant on $C_{i}$, then we can delete the $k$ rows and $\leq h+1$ columns to obtain a simple $(m-k)$-rowed matrix (deleting the $h$ columns non-constant on $C_{i}$ and possibly one extra column constant on $C_{i}$ and all 1 's on components above $C_{i}$ and all 0's on components if that column is present) and so by the Deletion Lemma 1.6 we are done if

$$
\frac{h+1}{k} \leq \frac{11}{4}
$$

From this we can assume there are no components of size $1,2,3$ since $h \leq 2^{k}-2$ for any $k$-rowed component.

It remains to show that each component in the graph of dotted edges is a clique. Assume that $C$ is a not a clique (in the dotted edge graph). We consider two cases.

Case 1. There is no pair of rows $(i, j)$ of $C$ which has the configuration $F_{0,7}$.

Assume the component has $k$ vertices and consider the $k$-rowed matrix formed from the possible non-constant columns on these $k$ rows. Let $h$ be the number of columns nonconstant on $C$. Using the Upper Bound Lemma 1.11 with $t=6$, the maximum number of columns non-constant on the component is at most

$$
\left\lfloor 2 k+\frac{2 k(k-1)}{4(k-2)}\right\rfloor
$$

But then for $k \geq 6$ and by (6), we are done. For $k=4$ and $k=5$ we must make more detailed arguments to eliminate the possibility $h=11$ for $k=4$ and the possibility $h=13$ for $k=5$.

For $k=4$ we deduce from the Upper Bound Lemma 1.11 proof that in order to have 11 non-constant columns we would need $\left[K_{4}^{1} K_{4}^{3}\right]$ and hence the component is a clique, a contradiction. For $k=5$ we deduce that in order to have 13 non-constant columns we would need $\left[K_{5}^{1} K_{5}^{4}\right]$ and three columns each with either two or three 1's or we have nine columns chosen from $\left[K_{5}^{1} K_{5}^{4}\right]$ and four columns each with either two or three 1 's. In either case, the component is a clique, a contradiction. 
Case 2. The rows of $C$ contain the configuration $F_{0,7}$.

Let $i, j$ be two rows of $C$ with the submatrix ${ }_{j}^{i}\left[\begin{array}{l}1 \\ 0\end{array}\right]$. We deduce that we do not have $i \cdots \cdots j$ and so we may assume $i \rightarrow j$ and we have the submatrix ${ }_{j}^{i}\left[\begin{array}{l}1111111 \\ 0000000\end{array}\right]$. But now it is true that for every other row $s$ we have either $i \rightarrow s$ or $s \rightarrow j$ since there will either be four 0's in row $s$ below the seven 1's yielding $i \rightarrow s$ or four 1's in row $s$ below the seven 0's yielding $s \rightarrow j$. Take the shortest path of dotted edges joining $i, j$ say $i=v_{1}, v_{2}, v_{3}, \ldots, v_{r}=j$ and with $v_{a}$ and $v_{a+1}$ joined by dotted edges for $1 \leq a \leq r-1$. Since either $v_{1} \rightarrow v_{a}$ or $v_{a} \rightarrow v_{r}$, we deduce that $r \geq 4$. One can verify using transitivity and the minimality of the path that $v_{s} \rightarrow v_{t}$ if $s+1<t$. As a sample note that with $r \geq 4$ then $r-1 \neq 2$ and so either $v_{1} \rightarrow v_{r-1}$ or $v_{r-1} \rightarrow v_{1}$ (the path was a shortest path). The latter is forbidden by $v_{1} \rightarrow v_{r}$ and $v_{r-1} \cdots v_{r}$ and transitivity. Now we can write down the $2 r-2$ possible non-constant columns on the $r$ rows $v_{1}, v_{2}, \ldots, v_{r}$ :

$$
\begin{aligned}
& \begin{array}{lllllllll}
a_{1}^{1} & a_{2}^{0} & a_{2}^{1} & a_{3}^{0} & a_{3}^{1} & \cdots & a_{r-1}^{0} & a_{r-1}^{1} & a_{r}^{0}
\end{array} \\
& \begin{array}{l}
v_{1} \\
v_{2} \\
v_{3} \\
v_{4} \\
v_{r-1} \\
v_{r}
\end{array} \quad\left(\begin{array}{ccccccccc}
0 & 1 & 1 & 1 & 1 & \ldots & 1 & 1 & 1 \\
1 & 0 & 0 & 1 & 1 & \ldots & 1 & 1 & 1 \\
0 & 0 & 1 & 0 & 0 & \ldots & 1 & 1 & 1 \\
0 & 0 & 0 & 0 & 1 & \ldots & 1 & 1 & 1 \\
\vdots & \vdots & \vdots & \vdots & \vdots & & \vdots & \vdots & \vdots \\
0 & 0 & 0 & 0 & 0 & \ldots & 0 & 0 & 1 \\
0 & 0 & 0 & 0 & 0 & \ldots & 0 & 1 & 0
\end{array}\right)
\end{aligned}
$$

where $a_{k}^{d}$ refers to the number of columns with a 0 in row $v_{k}$ and a $d$ in row $v_{k+1}$ with 1 's above and 0's below. We verify using $v_{s} \cdots v_{s+1}$ in the ordered set of rows $v_{1}, v_{2}, \ldots, v_{r}$ that

$$
1 \leq a_{s-1}^{1}+a_{s+1}^{0}+a_{s+1}^{1} \leq 3 \text { and } 1 \leq a_{s}^{1} \leq 3
$$

We may add up the inequalities $a_{s-1}^{1}+a_{s+1}^{0}+a_{s+1}^{1} \leq 3$ to obtain

$$
a_{1}^{1}+2 a_{2}^{1}+2 a_{3}^{1}+\cdots 2 a_{n-2}^{1}+a_{n-1}^{1}+a_{2}^{0}+a_{3}^{0}+\cdots+a_{r}^{0} \leq 3(r-1) .
$$

But using $a_{s}^{1} \geq 1$ for all possible $s$, yields

$$
a_{1}^{1}+a_{2}^{1}+a_{3}^{1}+\cdots a_{r-2}^{1}+a_{r-1}^{1}+a_{2}^{0}+a_{3}^{0}+\cdots+a_{r}^{0} \leq 2(r-1)+2 .
$$

Thus we can delete $r-1$ rows and $2(r-1)+2$ columns and obtain a simple matrix which satisfies the Deletion Lemma 1.6 for $r \geq 4$ meaning no such pair $i, j$ exists. This contradiction and the contradiction for Case 1 forces all components to be cliques and of size at least 4, establishing (iii).

Theorem 2.4 For $F_{1,4}=\left[\begin{array}{lllll}1 & 0 & 0 & 0 & 0 \\ 0 & 1 & 1 & 1 & 1\end{array}\right]$, and for $m \not \equiv 3(\bmod 4)$ and $m \geq 4$ we have

$$
\operatorname{forb}\left(m, F_{1,4}\right)=\left\lfloor\frac{11 m}{4}\right\rfloor+1
$$


and for $m \equiv 3(\bmod 4)$, we have

$$
\operatorname{forb}\left(m, F_{1,4}\right)=\left\lfloor\frac{11 m}{4}\right\rfloor
$$

Proof: In view of Lemma 2.2, we need only establish upper bounds for forb $\left(m, F_{1,4}\right)$. Our proof first handles the cases for $m \not \equiv 3(\bmod 4)$ and then proves the bound for $m \equiv 3(\bmod 4)$. We begin by establishing the bound $(7)$ for all $m \geq 3$. By Lemma 2.3, we know that the components of $D(A)$ can be assumed to be cliques of size at least 4 .

If a component of $k$ vertices $(k \geq 4)$ is a clique, then in any pair of rows of the clique, there is no configuration $F_{0,4}$. We apply Theorem 2.1 to obtain the maximum number of columns non-constant on the clique as $\left\lfloor 2 k+\frac{k}{2}\right\rfloor$. Now with

$$
\max \left\{\frac{1}{k}\left(\left\lfloor 2 k+\frac{k}{2}+1\right\rfloor\right): k \geq 4\right\} \leq \frac{11}{4}
$$

we may use (6) to establish the bound. This proves (7) for all $m$.

We now establish $(8)$ for $m \equiv 3(\bmod 4)$ by induction on $m$ using $(7)$. We verify the base case that for $m=3$, that forb $\left(3, F_{1,4}\right)=8=\left\lfloor\frac{11 \cdot 3}{4}\right\rfloor$. Now assume $m \equiv 3(\bmod 4)$ and $m \geq 7$. When applying induction for $m^{\prime}<m$ and $m^{\prime} \not \equiv 3(\bmod 4)$, we can only use forb $\left(m^{\prime}, F_{1,4}\right) \leq\left\lfloor\frac{11 m^{\prime}}{4}\right\rfloor+1$ from (7) which complicates the induction. Adapting the argument of the Deletion Lemma 1.6, we cannot delete $k$ rows and up to $\frac{11}{4} k-1$ columns from $A$ and obtain a simple matrix with no $F_{1,4}$. Adapting the argument of the Transitivity Lemma 1.7 we must have $2(p-1) \leq \frac{11}{4}-1$ to establish the appropriate version of $(1)$ and we must have $2(p-1)+(q-1) \leq 2\left(\frac{11}{4}\right)-1$ to establish the appropriate version of (2). Both are true and so we can establish the conclusions of the Transitivity Lemma 1.7. The Ordering Lemma 1.8 follows as before. Reorder the rows of $A$ to respect this order.

We now consider the components. If there is only one component then the result follows from the Upper Bound Lemma 1.11 with $t=6$. In this case we obtain $n \leq$ $\left\lfloor 2 m+\frac{2 m(m-1)}{4(m-2)}\right\rfloor \leq\left\lfloor\frac{11 m}{4}\right\rfloor$ for $m \geq 7$ which is assumed above. If there are two or more components with one of size $a$, then we deduce we must have a decomposition as in (5) with $a+b=m$. If $a \equiv 3(\bmod 4)$, we have $b \equiv 0(\bmod 4)$ and obtain the bound $\left\lfloor\frac{11 a}{4}\right\rfloor-2+\left(\left\lfloor\frac{11 b}{4}\right\rfloor+1\right)-2+3$ which yields the desired bound $\left\lfloor\frac{11 m}{4}\right\rfloor$. If $a \equiv 2(\bmod 4)$, we have $b \equiv 1(\bmod 4)$ and obtain the bound $\left(\left\lfloor\frac{11 a}{4}\right\rfloor+1\right)-2+\left(\left\lfloor\frac{11 b}{4}\right\rfloor+1\right)-2+3$ which yields the desired bound $\left\lfloor\frac{11 m}{4}\right\rfloor$. The remaining two congruences for $a$ follow in the same way.

It would be nice to apply the above arguments in Theorem 3.9 or in Theorem 3.14 to show that components are cliques. We cannot do so because we are using the stronger bound (4) from the Upper Bound Lemma 1.11 rather than (3) as is used in those theorems. 
Lemma 2.5 For $F_{1,5}=\left[\begin{array}{llllll}1 & 0 & 0 & 0 & 0 & 0 \\ 0 & 1 & 1 & 1 & 1 & 1\end{array}\right]$,

$$
\operatorname{forb}\left(m, F_{1,5}\right) \geq\left\lfloor\frac{15 m}{4}\right\rfloor+1 \text { for } m \equiv 0(\bmod 4) \text { and } m \geq 4 \text {. }
$$

Proof: We provide a construction for $A_{m}$, a simple $m \times\left\lfloor\frac{15 m}{4}\right\rfloor+1$ matrix which avoids the configuration $F_{1,5}$ for $m \geq 4$ and $m \equiv 0(\bmod 4)$. We can take $A_{4}=K_{4} . A_{m}$ can be constructed inductively as follows where $m-k \equiv 0(\bmod 4)$ :

$$
A_{m}=\left[\begin{array}{cc}
A_{m-4}-\left[\begin{array}{c}
1 \\
\vdots \\
1
\end{array}\right] & 1^{\prime} s \\
0^{\prime} s & A_{4}
\end{array}\right] .
$$

Hence, we have forb $\left(m, F_{1,5}\right) \geq\left\lfloor\frac{15 m}{4}\right\rfloor+1$ for $m \geq 4$ and $m \equiv 0(\bmod 4)$.

We can construct suitable $A_{5}, A_{6}, A_{7}$ as base cases and then use the construction (9) but these constructions unfortunately miss the bound by a small constant (up to 3 ). The analysis for the cases $m \not \equiv 0(\bmod 4)$ will require too much detail for this paper but would follow in the spirit of the argument for Theorem 2.4 for the case $m \equiv 3(\bmod 4)$.

We now show forb $\left(m, F_{1,5}\right) \leq \frac{15 m}{4}+1$ following the proof of Theorem 2.4 . It is easily verified for $m=1,2,3,4$. Assume $m \geq 5$ and proceed by induction. Let $A$ be a simple $m \times n$ matrix with no configuration $F_{1,5}$. We wish to show $n \leq \frac{15 m}{4}+1$.

Lemma 2.6 Our structure $D(A)$ can be assumed to have the following properties.

(i) Each pair of rows is connected by exactly one edge, directed or dotted.

(ii) The graph on the directed edges is transitive and contains no cycles.

(iii) All components of the graph on the dotted edges are cliques of size at least 5.

Proof: In view of our bound $\frac{15 m}{4}+1$ i.e. $c=15 / 4$ with $p=1, q=5$, we have (i) and (ii) by the Transitivity Lemma 1.7. As before, the components induced by the dotted edges form components can be ordered by the Ordering Lemma 1.8. Reorder the rows of $A$ to respect that order. We proceed as in the proof of Lemma 2.3. With $p=1$, we have that for two rows $x, y$ with $x \in C_{i}$ and $y \in C_{j}$ and $i<j$ (in the ordering), then $x \rightarrow y$ and so there is no submatrix $\begin{aligned} & x \\ & y\end{aligned}\left[\begin{array}{l}0 \\ 1\end{array}\right]$ as indicated on rows $x, y$. If we have a component $C_{i}$ on $k$ vertices with $h$ columns non-constant on $C_{i}$, then we can delete the $k$ rows and $h+1$ columns (one extra column may be required since there may be two columns, one with all 0's on $C_{i}$ or all 1's on $C_{i}$ each canonical with respect to $C_{i}$ (using Definition 1.9) and have a simple $(m-k)$-rowed matrix and so by the Deletion Lemma 1.6 we are done if

$$
\frac{h+1}{k} \leq \frac{15}{4}
$$

From this we can assume there are no components of size $1,2,3,4$ using $h \leq 2^{k}-2$. 
We wish to show that each component $C$ on 5 or more vertices is a clique. Assume that $C$ is a not a clique (in the dotted edge graph)

Case 1. In the component $C$ there is no pair of rows $i, j$ which has the configuration $F_{0,9}$.

Note this is possible and still have $i \rightarrow j$. Assume the component has $k$ vertices and consider the $k$-rowed matrix formed from the possible non-constant columns on these $k$ rows. Assume that any pair of rows $i, j$ has at most 8 configurations $\left[\begin{array}{l}0 \\ 1\end{array}\right]$ and so by the Upper Bound Lemma 1.11, the maximum number of columns non-constant on $C$ is

$$
\left\lfloor 2 k+\frac{4 k(k-1)}{4(k-2)}\right\rfloor .
$$

But then for $k \geq 5$ and by (10), we deduce that such a $C$ does not occur and so Case 1 does not occur.

Case 2. In the component $C$ there are two rows $i, j$ which have at least 9 columns with the configuration ${ }_{j}^{i}\left[\begin{array}{l}1 \\ 0\end{array}\right]$.

We deduce that we do not have $i \cdots j$ and so we may assume $i \rightarrow j$ and we have the submatrix ${ }_{j}^{i}\left[\begin{array}{l}111111111 \\ 00000000\end{array}\right]$. But now it is true that for every other row $s$ we have either $i \rightarrow s$ or $s \rightarrow j$ since there will either be five 0's in row $s$ below the nine 1's yielding $i \rightarrow s$ or five 1 's in row $s$ below the nine 0's yielding $s \rightarrow j$. Following Lemma 2.3 we take the shortest path of dotted edges joining $i, j: i=v_{1}, v_{2}, v_{2}, \ldots, v_{r}=j$ and consider the $2 r-2$ possible non-constant columns on the $r$ rows $v_{1}, v_{2}, \ldots, v_{r}$. Then we can delete $r-1$ rows and $3(r-1)+2$ columns and obtain a simple matrix which satisfies the Deletion Lemma 1.6 for $r \geq 4$ meaning no such pair $i, j$ exists and so Case 2 does not occur.

Thus components of size at least 5 are cliques. This establishes (iii).

Theorem 2.7 For $F_{1,5}=\left[\begin{array}{llllll}1 & 0 & 0 & 0 & 0 & 0 \\ 0 & 1 & 1 & 1 & 1 & 1\end{array}\right]$,

$$
\text { forb }\left(m, F_{1,5}\right) \leq\left\lfloor\frac{15 m}{4}\right\rfloor+1 \text { with equality for } m \geq 4 \text { and } m \equiv 0(\bmod 4) \text {. }
$$

Proof: In view of Lemmas 2.5, 2.6, we need only establish the upper bound with the graph on dotted edges from $D(A)$ consisting of cliques of size at least 5 . If a component of $k$ vertices $(k \geq 5)$ is a clique, then there are at most 8 configurations $\left[\begin{array}{l}0 \\ 1\end{array}\right]$ in any pair of rows and so by the Upper Bound Lemma 1.11, the maximum number of columns non-constant on the clique is $\left\lfloor 2 k+\frac{4 k(k-1}{4(k-2)}\right\rfloor$. Using

$$
\max _{k: k \geq 5} \frac{1}{k}\left\lfloor 2 k+\frac{4 k(k-1)}{4(k-2)}+1\right\rfloor \leq \frac{15}{4},
$$

we establish the bound using (10).

It is frustrating that some of these arguments fail for $F_{1, q}$ for larger $q$. The argument given does not immediately show components are cliques for $q \geq 6$. Our general construction in the final section has clique components. 


\section{Exact Bounds for $F_{2,5}$ and $F_{2,4}$}

We begin with exact bounds for $F_{2,5}$ followed by $F_{2,4}$, the latter case being more difficult. Both arguments are rather delicate, perhaps because there are a number of constructions achieving the bounds.

Lemma 3.1 For $F_{2,5}=\left[\begin{array}{ccccccc}1 & 1 & 0 & 0 & 0 & 0 & 0 \\ 0 & 0 & 1 & 1 & 1 & 1 & 1\end{array}\right]$

$$
\text { forb }\left(m, F_{2,5}\right) \geq 4 m \text { for } m=4,8 \text { and } m \geq 11 \text {. }
$$

Proof: We provide constructions for $A_{m}$, a simple $m \times 4 m$ matrix which avoids the configuration $F_{2,5}$ for the specified values of $m$. We can take $A_{4}=K_{4}$. $A_{m}$ can be constructed inductively from $A_{m-4}$ using

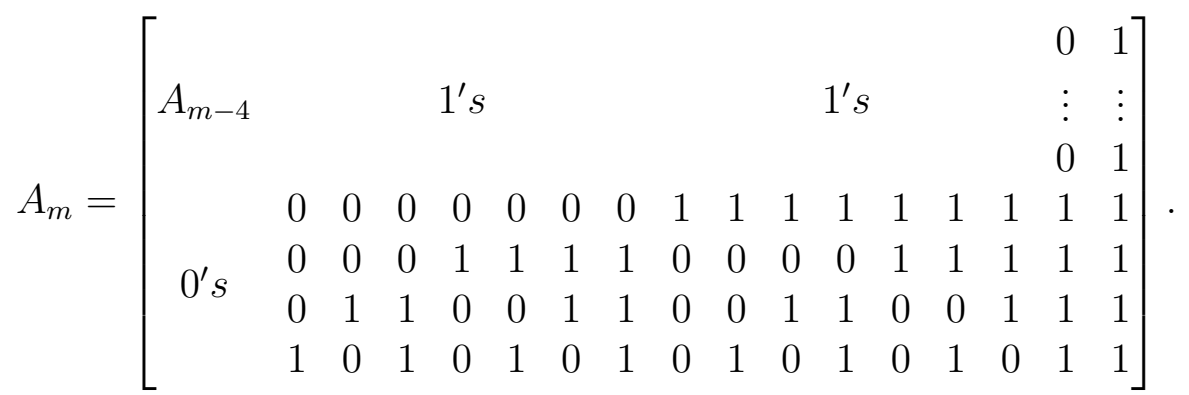

We need constructions for $A_{m}$ when $m \equiv 1,2,3(\bmod 4)$. For two matrices $A, B$ we use the notation $A-B$ given in Definition 1.3. If $a+b+c=m, b \geq 3$, we can construct $A_{m}$ recursively as follows:

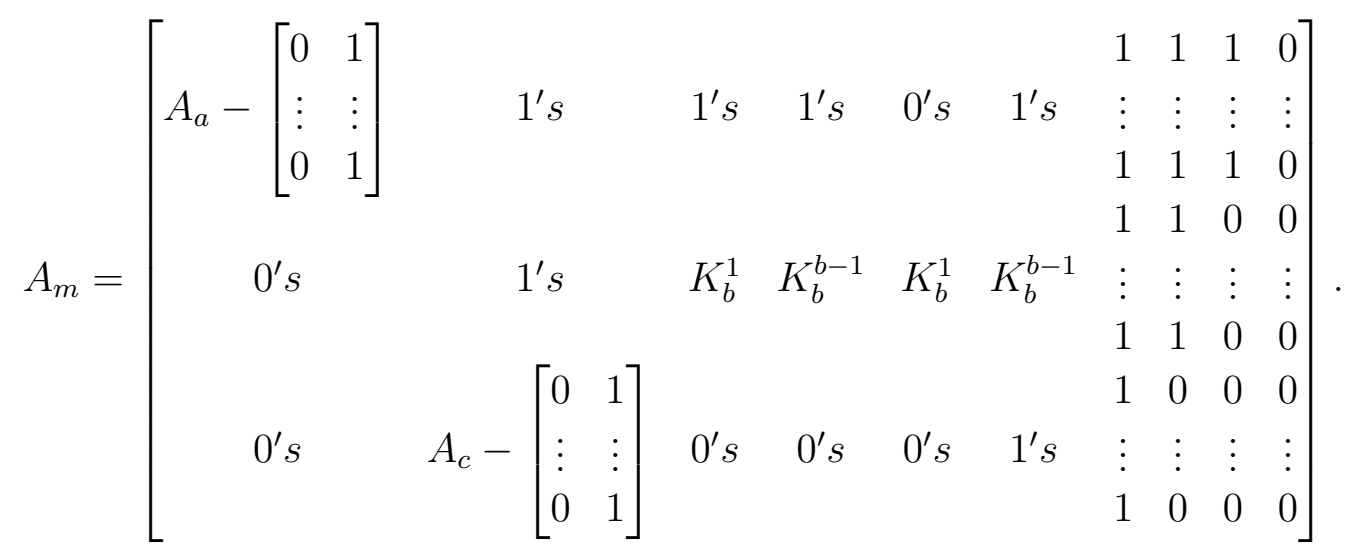

Hence, we have forb $\left(m, F_{2,5}\right) \geq 4 m$ for $m=4,8$ and $m \geq 11$ (e.g. using $a=c=4$ ).

We show forb $\left(m, F_{2,5}\right) \leq 4 m$ by induction. It is true for $m=1,2,3,4$. Assume $m \geq 5$ and proceed by induction. Let $A$ be a simple $m \times n$ matrix with no configuration $F_{2,5}$. We wish to show $n \leq 4 m$. 
Lemma 3.2 Our structure $D(A)$ can be assumed to have the following properties.

(i) Each pair of rows is connected by exactly one edge, directed or dotted.

(ii) The graph on the directed edges is transitive and contains no cycles.

(iii) All components of the graph on the dotted edges are cliques or possibly a three vertex component of two dotted edges.

Proof: We construct $D(A)$ and, in view of our bound of $4 m$, we have the following properties (i) and (ii) by the Transitivity Lemma 1.7 with $c=4$ and $p=2, q=5$. Property (iii) is more work.

Assuming that there exists a component which is not a clique, there must be three rows $i, j, k$ with $i \longrightarrow j, k \cdots j, k \cdots i$ (hence $i, j, k$ are in the same component). Let us analyze this in detail. The possible entries for these rows are

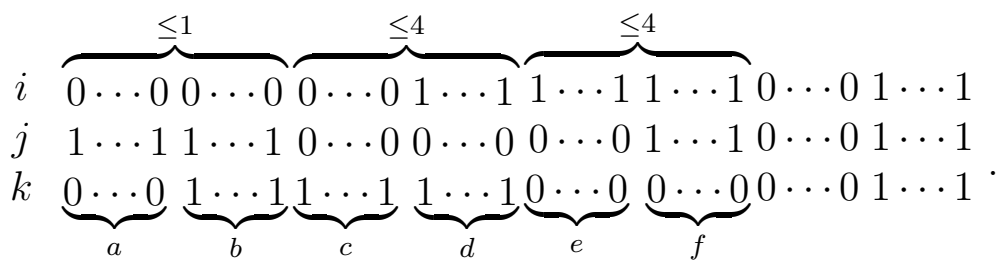

The total number of non-constant columns in these three rows is given by $\alpha=a+b+c+$ $d+e+f$. Based on the edge $k \cdots i$ we have $2 \leq b+c \leq 4,2 \leq e+f \leq 4$ and based on the edge $k \cdots j$ we have $2 \leq a+f \leq 4,2 \leq c+d \leq 4$, and based on the edge $i \rightarrow j$ we have $a+b \leq 1$. We deduce that $\alpha \leq 9$. We note that we could eliminate rows $j$ and $k$ and the $\alpha$ columns and the resulting matrix would be simple. Thus by our Deletion Lemma 1.6, we may assume $\alpha \geq 9$. Thus, the only possibility is $\alpha=9$ implying

$$
a+b=1, \quad c+d=4 \text { and } e+f=4 .
$$

By our Deletion Lemma 1.6, removing a row must force removing 5 or more columns in order to have the resulting matrix to be simple. If we wish to remove row $i$, then we can force the resulting $(m-1)$-rowed matrix to be simple by deleting the columns associated with $e$ and $b$ and $(c$ or $d$ ) and ( $a$ or $f$ ). Thus, $a+b+e+\min \{c, d\} \geq 5$, yielding

$$
e+\min \{c, d\} \geq 4 \text {. }
$$

Removing row $j$ and the following combination of columns $d$ and $a$ and (e or $f$ ) and ( $b$ or $c$ ) results in a simple matrix. Thus, $a+b+d+\min \{e, f\} \geq 5$ yielding

$$
d+\min \{e, f\} \geq 4 .
$$

Removing row $k$ and the following combination of columns $d$ and $e$ and ( $a$ or $b)$ and (c or $f$ ) results in a simple matrix. Thus, $d+e+\min \{a, b\}+\min \{c, f\} \geq 5$ and $\min \{a, b\}=0$ yielding

$$
d+e+\min \{c, f\} \geq 5 .
$$

We note $c+d=4$ implies $\min \{c, d\} \leq 2$ and similarly $\min \{e, f\} \leq 2$. In addition, $b+c \geq 2$ and $a+f \geq 2$. The only feasible solution is to have $c=d=e=f=2$ and 
either $a=1$ or $b=1$. Thus if $i, j, k$ did induce a component of size 3 then we can use this information for this 'special' component.

We now consider the components in the graph formed by the dotted edges. We first note that any components of size $k=1,2,3$ must be a clique of dotted edges or our special component of two dotted edges on three vertices that was analyzed above. Suppose we have a component, not a clique, with 4 or more vertices. There must be at least two vertices at distance two. Assume the two vertices $i, j$ have a shortest path $i, k, j$ of dotted edges joining them. There must be a directed edge connecting $i$ and $j$. Since the two are interchangeable, the following configuration takes place:

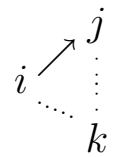

Let $l$ be any other vertex of the component and on the four rows we have the situation below. We will arrive at a contradiction.

$\begin{array}{cccccccc}i & 0 & 00 & 11 & 11 & 11 & 0 \cdots 0 & 1 \cdots 1 \\ j & 1 & 00 & 00 & 00 & 11 & 0 \cdots 0 & 1 \cdots 1 \\ k & 0 \text { or } 1 & 11 & 11 & 00 & 00 & 0 \cdots 0 & 1 \cdots 1 \\ l & L & C & D & E & F & G & H\end{array}$

The matrices $C, D, E, F$ are size $1 \times 2$ and $L$ is size $1 \times 1$ and $G, H$ are 1 -rowed matrices. We note that if $\# 1^{\prime} s(G)+\# 0^{\prime} s(H) \leq 3$, using Definition 1.4, then we could delete the 3 rows $i, j, k$ and the up to 12 columns non-constant on rows $i, j, k, l$ to obtain a simple matrix, violating the Deletion Lemma 1.6. We conclude

$$
\# 1^{\prime} s(G)+\# 0^{\prime} s(H) \geq 4 .
$$

We now establish that the following five cases do not occur.

Case 1. The edges $i \cdots \cdot l, j \cdots \cdot l, k \cdots \cdot l$ are all present.

Applying our previous result to the triple $(i, j, l)$, we deduce $\# 1^{\prime} s(C G)=2$ and $\# 0^{\prime} s(F H)=2$. But then by $(13), \# 1^{\prime} s(G)=2$ and $\# 0^{\prime} s(H)=2$ and hence $\# 1^{\prime} s(F)=2$ and $\# 0^{\prime} s(C)=2$. Using $k \cdots \cdot l$, we deduce that $\# 0^{\prime} s(L C D H) \leq 4$ and so $\# 0^{\prime} s(L D)=0$ and so $\# 0^{\prime} s(D)=0$. Similarly $\# 1^{\prime} s(L E F G) \leq 2$ and then $\# 0^{\prime} s(E)=2$. Now we could delete row $i$ and the up to three columns in $L, E$ and still preserve a simple matrix. Thus Case 1 does not occur.

Case 2. The edges $i \cdots \cdots l, j \cdots \cdots l, k \rightarrow l$ are all present.

Using $k \rightarrow l$, we have $\# 1^{\prime} s(E F G) \leq 1$ and in particular $\# 1^{\prime} s(F) \leq 1$. But by the same logic at the beginning of Case 1 , we obtain $\# 1^{\prime} s(F)=2$, a contradiction. Thus Case 2 does not occur.

Case 3. The edges $i \rightarrow l, k \cdots \cdot l$ are both present.

Using $i \rightarrow l$, we have $\# 1^{\prime} s(L C G) \leq 1$ and so by $(13)$, $\# 0^{\prime} s(H) \geq 3$. Now using the previous argument on the triple $(i, l, k)$, we deduce that $\# 0^{\prime} s(D H)=2$, a contradiction. Thus Case 3 does not occur. 
Case 4. The edges $i \rightarrow l, j \cdots . . l, k \rightarrow l$ are all present.

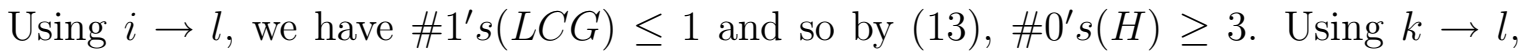
we have $\# 1^{\prime} s(E F G) \leq 1$. Using $j \cdots . . . l$, we have $\# 0^{\prime} s(L F) \leq 1$. Note that this yields $\# 1^{\prime} s(F) \leq 1$ and $\# 0^{\prime} s(F) \leq 1$ and so $\# 1^{\prime} s(F)=1$ and $\# 0^{\prime} s(F)=1$. Now $\# 0^{\prime} s(L F) \leq 1$ implies $\# 0^{\prime} s(L)=0$ and hence \# $1^{\prime} s(L)=1$. Now \# $1^{\prime} s(L E F G) \leq 1$ implies $\# 1^{\prime} s(G)=0$ which implies $\# 0^{\prime} s(H) \geq 4$ by (13) which then forces $\# 0^{\prime} s(L F H) \geq 5$ which contradicts that $j \cdots . . . l$. Thus Case 4 does not occur.

Case 5. The edges $i \cdots \cdot l, l \rightarrow j, k \cdots \cdot l$, are all present.

This case is covered by Case 3 by using the fact that $A$ does not have the configuration $F_{2,5}$ if and only if $A^{c}$ (the $(0,1)$-complement of $A$ ) does not have the configuration $F_{2,5}^{c}$ where $F_{2,5}$ and $F_{2,5}^{c}$ are the same configurations. Thus Case 5 does not occur.

We are going to show that either we have the three directed edges $i \rightarrow l, j \rightarrow l, k \rightarrow l$ or the three directed edges $l \rightarrow i, l \rightarrow j, l \rightarrow k$. We use the five cases above in our argument.

If $l \rightarrow i$, we will have $l \rightarrow j$ based on transitivity. Case 3 (with the roles of $i$ and $l$ interchanged) eliminates $l \cdots k$ and transitivity forces that $k \rightarrow l$ cannot happen since we do not have $k \rightarrow i$. Thus we must have $l \rightarrow k$ (by property (i)) which means all edges from $l$ are directed.

If $l \cdots i$ then transitivity ensures $j \rightarrow l$ does not happen since that would have forced $i \rightarrow l$. Cases 1 and 2 eliminate having $l \cdots j$. The remaining possibility is $l \rightarrow j$ which then prevents having $k \rightarrow l$. Case 4 eliminates the possibility of $l \rightarrow k$ and Case 5 eliminates the possibility $k \cdots \cdots l$. Transitivity does not allow $k \rightarrow l$ and so the case $l \cdots i$ cannot occur.

If $i \rightarrow l$ then transitivity forces $l \rightarrow k$ to not occur. Case 3 eliminates the possibility of having $l \cdots \cdots k$. Now, if $k \rightarrow l$, then because transitivity would forbid $l \rightarrow j$ and Case 4 would forbid $l \cdots j$ we would have $j \rightarrow l$ and so all edges from $i, j, k$ are directed into $l$.

As promised, we have shown for any other vertex $l$ of the same component that there are no dotted edges joining $l$ to $i, j, k$ for any vertex $l$ in the same component. This contradicts that $i, j, k$ is in a component (of the graph of dotted edges) with other vertices. We have established property (iii).

We will now describe a general argument used in the proofs of Theorem 3.9 and Theorem 3.14 for the forbidden configurations $F_{2, q}$ for $q=4,5$. Assume we are considering an $m \times n$ simple matrix $A$ with no configuration $F_{2, q}$. Assume that the properties (i),(ii),(iii) of Lemma 3.2 (or Lemma 3.11) hold and that we have reordered the rows of $A$ by the order from the Ordering Lemma 1.8. Let $m^{\prime}$ denote the number of components and let $c_{k}$ be the number of components which have $k$ vertices.

We are going to establish bounds for the number of columns in $A$ by separately considering varied columns ( $t$-varied for $t \geq 1)$ and the flat columns (0-varied) defined in Definition 1.10. The following are two easy bounds that prepare us for the more careful bounds of Claims 3.5 and 3.7 .

Claim 3.3 Let $q=4$ or 5 . The number of varied columns of $A$ is at most

$$
\sum_{C_{i}:\left|C_{i}\right| \geq 2}(q-1)\left|C_{i}\right|=(q-1)\left(m-c_{1}\right) .
$$


Proof: Consider a component $C_{i}$ with $\left|C_{i}\right|$ vertices. For $\left|C_{i}\right| \geq 2$ and the component being a clique, the presence of dotted edges means we avoid the configuration $F_{0, q}$ on the clique. By the Upper Bound Lemma 1.11 with $t=2(q-1)$ in (3), there can be no more than $(q-1)\left|C_{i}\right|$ columns which are non-constant on these $\left|C_{i}\right|$ rows. In fact, if $\left|C_{i}\right|=1$, then there are no such columns. There are $m-c_{1}$ rows of $A$ in components of size at least 2. In the cases $F_{2, q}$ for $q=4,5$ it is possible there are components of size 3 which are not cliques but special arguments bound the number of columns non-constant on such a component as 9 if $q=5$ and as 7 if $q=4$. In both cases the bounds are less than $(q-1)\left|C_{i}\right|$. Note that $\sum_{i:\left|C_{i}\right| \geq 2}\left|C_{i}\right|=m-c_{1}$. Thus the total number of varied columns is at most

$$
\sum_{C_{i}:\left|C_{i}\right| \geq 2}(q-1)\left|C_{i}\right|=(q-1)\left(m-c_{1}\right) .
$$

Claim 3.4 The number of flat columns is at most $2 m^{\prime}$.

Proof: We note that in view of the component ordering from the Ordering Lemma 1.8, the flat columns of $A$ avoid the submatrix $\left[\begin{array}{ll}0 & 0 \\ 1 & 1\end{array}\right]$. There are at most $m^{\prime}+1$ columns with no submatrix $\left[\begin{array}{l}0 \\ 1\end{array}\right]$. corresponding to the flat columns which have all 1 's on some initial set (possible empty) of components $C_{1}, C_{2}, \ldots, C_{i}$ and all 0's on the remaining components $C_{i+1}, C_{i+2}, \ldots, C_{m^{\prime}}$. If we have a flat column of $A$ with a pair $i<j$ of components where the column is 0 's on $C_{i}$ and 1's on $C_{j}$, then there is a pair $(k, k+1)$ with the column having 0's on the rows of component $C_{k}$ and 1's on the rows of component $C_{k+1}$. There are only $m^{\prime}-1$ pairs $k, k+1$ and so at most $m^{\prime}-1$ flat columns of $A$ which have the submatrix $\left[\begin{array}{l}0 \\ 1\end{array}\right]$. Thus $A$ has at most $2 m^{\prime}$ flat columns.

Given that any column is either varied or flat, we can use Claims 3.3 and 3.4 to deduce:

$$
n \leq(q-1)\left(m-c_{1}\right)+2 m^{\prime}=2 c_{1}+\sum_{k \geq 2}((q-1) k+2) c_{k} .
$$

We now proceed to more carefully analyze the number of columns and show we have overcounted in Claims 3.3 and 3.4.

We first improve on Claim 3.3. Define

$$
v_{i, t}=\text { number of } t \text {-varied columns non-constant on } C_{i} .
$$

We deduce that the number of varied columns is

$$
\sum_{i} \sum_{t \geq 1} \frac{1}{t} v_{i, t}
$$

For a component $C_{i}$ we define $r_{i}$ to be

$$
r_{i}=(q-1)\left|C_{i}\right|-\sum_{t} \frac{1}{t} v_{i, t},
$$


or more usefully

$$
r_{i}=\left((q-1)\left|C_{i}\right|-\sum_{t \geq 1} v_{i, t}\right)+\sum_{t \geq 1} \frac{t-1}{t} v_{i, t}=r_{i}^{(1)}+r_{i}^{(2)},
$$

where

$$
r_{i}^{(1)}=\left((q-1)\left|C_{i}\right|-\sum_{t \geq 1} v_{i, t}\right)
$$

and (using the notation $n(\alpha)$ from Definition 1.10),

$$
r_{i}^{(2)}(\alpha)=\frac{n(\alpha)-1}{n(\alpha)} \quad \text { and } \quad r_{i}^{(2)}=\sum_{\alpha} \frac{n(\alpha)-1}{n(\alpha)}
$$

where we are summing over columns $\alpha$ which are non-constant on $C_{i}$. Note that the expression $r_{i}^{(1)}$ counts the difference between $(q-1)\left|C_{i}\right|$ (which is the maximum number of columns non-constant on $C_{i}$ ) and the actual number. Also note that $r_{i}^{(2)}$ can be computed column by column. Define

$$
r=\sum_{i=1}^{m^{\prime}} r_{i}
$$

Using (15), we deduce the following.

Claim 3.5 Let $q=4$ or 5 . The number of varied columns of $A$ is at most

$$
\sum_{C_{i}:\left|C_{i}\right| \geq 2}(q-1)\left|C_{i}\right|-r=(q-1)\left(m-c_{1}\right)-r .
$$

The value of Claim 3.5 is that we can estimate $r$ by estimating $r_{i}$, component by component and column by column and we will do so using (16).

The bound on the number of flat columns in Claim 3.4 can be sharpened by noticing that varied columns can interfere by containing a 0 on a row of component $C_{k}$ and a 1 on a row of component $C_{k+1}$ for some $k$. Given a varied column which is non-constant on $C_{i}$, then it is either canonical with respect to $C_{i}$ or it has at least one 0 in a component above $C_{i}$ or at least one 1 in a component below $C_{i}$. Thus for any non-canonical column, there exists a $k$ such that the column contains a 0 on a row of component $C_{k}$ and a 1 on a row of component $C_{k+1}$. In particular, among the columns of $A$ which are equal to a given non-constant $\left|C_{i}\right| \times 1$ column $\alpha$ on $C_{i}$, at most one is canonical with respect to $C_{i}$ and the rest are non-canonical with respect to $C_{i}$. This idea drives Claim 3.7 that follows. Non-canonical varied columns will drive down the bound for the number of flat columns. Note that $t$-varied columns for $t \geq 2$ are always non-canonical with respect to any component.

As noted in Claim 3.4, there are at most $m^{\prime}+1$ flat columns which do not contain the submatrix $\left[\begin{array}{l}0 \\ 1\end{array}\right]$ and there are at most $m^{\prime}-1$ flat columns which contain the submatrix 
$\left[\begin{array}{l}0 \\ 1\end{array}\right]$, each containing for some index $k$, all 0's on component $C_{k}$ and all 1's on $C_{k+1}$. Other columns, in particular non-canonical columns, may interfere with this count because for each $k$ and each choice of row $a$ in $C_{k}$ and each choice of row $b$ in $C_{k+1}$ there is at most one column in $A$ with a 0 on row $a$ and a 1 on row $b$ by the Ordering Lemma 1.8. Let $\alpha$ be a non-canonical varied column of $A$. Define $s^{\prime}(\alpha)$ to be the sum over all $i$ of the sum over all pairs of entries $(a, b)$, where $a$ is a row of $C_{i}$ and $b$ is a row of $C_{i+1}$ and $\alpha$ has a 0 on row $a$ and a 1 on row $b$, of the value $\frac{1}{\left|C_{i}\right|\left|C_{i+1}\right|}$. Define $s^{\prime}$ as the sum over non-canonical varied columns $\alpha$ :

$$
s^{\prime}=\sum_{\alpha} s^{\prime}(\alpha)
$$

Claim 3.6 The number of flat columns is at most $2 m^{\prime}-\left\lceil s^{\prime}\right\rceil$.

Proof: As noted in Claim 3.4, the number of flat columns which do not contain the submatrix $\left[\begin{array}{l}0 \\ 1\end{array}\right]$ (respecting the row order) is at most $m^{\prime}+1$. For each $k$ with $1 \leq k \leq m^{\prime}-1$, the total number of row pairs $(a, b)$ where $a$ is a row of $C_{k}$ and $b$ is a row of $C_{k+1}$ is $\left|C_{k}\right|\left|C_{k+1}\right|$. Thus $\left\lceil s^{\prime}\right\rceil$ provides a lower bound for the number of indices $k, 1 \leq k \leq m^{\prime}-1$, for which some varied column has a 0 on some row of $C_{k}$ and a 1 on some row of $C_{k+1}$ and hence for which no flat column has all 0's on component $C_{k}$ and all 1 's on $C_{k+1}$. Thus the number of flat columns which contain $\left[\begin{array}{l}0 \\ 1\end{array}\right]$ is at most $m^{\prime}-1-\left\lceil s^{\prime}\right\rceil$.

One could be more precise since if we have a varied column with even one pair of entries with a 0 in a component $C_{i}$ and 1 in $C_{i+1}$ then there is no flat column which has such a pair of entries (or vice-versa) and so we can reduce the bound on the number of flat columns which contain $\left[\begin{array}{l}0 \\ 1\end{array}\right]$ by 1 . We don't attempt this here.

We wish to compute an estimate for $s^{\prime}$, component by component, and so we compute $s=\sum_{i=1}^{m^{\prime}} s_{i}$ where $s_{i}$ is defined below. First define

$$
c_{\max }=\max _{k: 1 \leq k \leq m^{\prime}}\left|C_{k}\right| .
$$

For a component $C_{i}$, and for each non-canonical column $\alpha$, that is non-constant on $C_{i}$, we compute a number $s_{i}(\alpha)$ by one of the two expressions below:

If $\alpha$ is $t$-varied for $t \geq 2$, and also non-constant on other components then set

$$
s_{i}(\alpha)=\frac{1}{2 c_{\max }^{2}}
$$

If $\alpha$ is 1 -varied (only non-constant on $C_{i}$ ), then set

$$
s_{i}(\alpha)=\frac{1}{\left|C_{i}\right|}
$$

We then define $s_{i}=\sum_{\alpha} s_{i}(\alpha)$ where the sum is over all non-canonical varied columns $\alpha$ that are non-constant on $C_{i}$ and define $s=\sum_{i=1}^{m^{\prime}} s_{i}$. 
Claim 3.7 The number of flat columns is at most $2 m^{\prime}-\lceil s\rceil$. We have $s \leq s^{\prime}$.

Proof: We show $s \leq s^{\prime}$ and use Claim 3.6. Consider a non-canonical varied column $\alpha$ of $A$. First assume $\alpha$ is $t$-varied for some $t \geq 2$ and in particular is non-constant on $C_{i_{1}}, C_{1_{2}}, \ldots, C_{i_{t}}$. Consider $C_{i_{j}}$ and $C_{i_{j+1}}$ for $1 \leq j \leq t-1$. If $i_{j}+1=i_{j+1}$, then there is at least one pair of rows $(a, b)$ with $a$ in $C_{i_{j}}$ and $b$ in $C_{i_{j+1}}$ so that the column has a 0 in row $a$ and $a 1$ in row $b$. This yields a contribution to $s^{\prime}(\alpha)$ of $1 /\left(\left|C_{i_{j}}\right|\left|C_{i_{j+1}}\right|\right)$. If $i_{j}+1<i_{j+1}$, then there will be some index $k$ with $i_{j} \leq k<i_{j+1}$ where $a$ in $C_{k}$ and $b$ in $C_{k+1}$ so that $\alpha$ has a 0 in row $a$ and a 1 in row $b$. This yields a contribution to $s^{\prime}(\alpha)$ of $1 /\left(\left|C_{k}\right|\left|C_{k+1}\right|\right)$ where $1 /\left(\left|C_{k}\right|\left|C_{k+1}\right|\right) \geq 1 / c_{\max }^{2}$. We deduce that $s^{\prime}(\alpha) \geq \frac{n(\alpha)-1}{c_{\max }^{2}}$. We now check that

$$
\sum_{j: 1 \leq j \leq t} s_{i_{j}}(\alpha)=t \cdot \frac{1}{2 c_{\max }^{2}} \leq \frac{t-1}{c_{\max }^{2}} \leq s^{\prime}(\alpha)
$$

using $t / 2 \leq t-1$ for $t \geq 2$.

Second, assume $\alpha$ is 1-varied and non-constant on $C_{i}$ and so constant on other components. You might note that if there is only one component, then every varied column is canonical and so $s=0$. If $\alpha$ is all 0 's on $C_{i-1}$ then we have a 0 on any row of $C_{i-1}$ and a 1 on some row of $C_{i}\left(\alpha\right.$ is non-constant on $\left.C_{i}\right)$ and so $s^{\prime}(\alpha) \geq \frac{1}{\left|C_{i}\right|}$. We can do the same if $C_{i+1}$ is all 1's. If neither of these cases occur then, given that $\alpha$ is non-canonical, $\alpha$ has an index $j$ so that $\alpha$ is all 0 's on $C_{j}$ and all 1 's on $C_{j+1}$ yielding $s^{\prime}(\alpha) \geq 1$. In any case, $s_{i}(\alpha) \leq s^{\prime}(\alpha)$.

Summing over all non-canonical varied columns $\alpha$, We deduce that $s<s^{\prime}$.

This count for $s_{i}$ could result in $s<s^{\prime}$ but the intent of defining the $s_{i}$ 's is to allow us to consider the components (and columns) separately in our proof. We note the following:

Remark 3.8 A non-canonical column $\alpha$ non-constant on $C_{i}$ always has $s_{i}(\alpha)>0$.

Note that $s \leq s^{\prime} \leq\left\lceil s^{\prime}\right\rceil \leq m^{\prime}-1$.

We have refined (14) using Claims 3.5, 3.6 into

$$
n \leq(q-1)\left(m-c_{1}\right)+2 m^{\prime}-(r+s)=2 c_{1}-(r+s)+\sum_{k \geq 2}((q-1) k+2) c_{k} .
$$

Our proofs of Theorems 3.9 and 3.14 show that $r+s$ is large enough to obtain the exact bound for $n$.

Theorem 3.9 For $F_{2,5}=\left[\begin{array}{lllllll}1 & 1 & 0 & 0 & 0 & 0 & 0 \\ 0 & 0 & 1 & 1 & 1 & 1 & 1\end{array}\right]$,

$$
\operatorname{forb}\left(m, F_{2,5}\right) \leq 4 m \text { with equality for } m=4,8 \text { and } m \geq 11
$$


Proof: In view of Lemma 3.1 we need only establish the upper bound and in view of Lemma 3.2 we may assume all components are cliques or the special component on 3 vertices. As indicated, we use the Ordering Lemma 1.8 to order the components and then follow the general argument given above.

Using Claims 3.5 and 3.7 together, $A$ has a maximum of $4\left(m-c_{1}\right)+2 m^{\prime}-(r+s)$ columns. From (14) we note

$$
4\left(m-c_{1}\right)+2 m^{\prime}=2 c_{1}+\sum_{k \geq 2}(4 k+2) c_{k}=2 c_{1}+10 c_{2}+14 c_{3}+18 c_{4}+\cdots
$$

We will show below that

$$
r+s \geq \sum_{k \geq 2} 2 c_{k} .
$$

But then by (21)

$$
n \leq 2 c_{1}+\sum_{k \geq 2}(4 k+2) c_{k}-\sum_{k \geq 2} 2 c_{k}=2 c_{1}+4 \sum_{k \geq 2} k c_{k} \leq 4 m .
$$

We will verify (23) by showing that each component $C_{i}$ with $\left|C_{i}\right| \geq 2$ has $r_{i}+s_{i} \geq 2$. Consider a non-canonical column $\alpha$ which is non-constant on $C_{i}$. If the column is $t$-varied, for $t \geq 2$ then

$$
\text { (i) } s_{i}(\alpha)>0, \quad r_{i}^{(2)}(\alpha)=\frac{t-1}{t} \geq \frac{1}{2} .
$$

If the column is 1-varied,

$$
\text { (ii) } \quad s_{i}(\alpha)=\frac{1}{\left|C_{i}\right|}, \quad r_{i}^{(2)}(\alpha)=0
$$

These follow from (19) and (20). Assume $C_{i}$ is a component of size $k \geq 2$. The Upper Bound Lemma 1.11 ensures that there are at most $4 k$ columns non-constant on $C_{i}$. We consider three cases and in each case we verify that $r_{i}+s_{i} \geq 2$.

Case 1. The number of columns non-constant on $C_{i}$ is $\leq 4 k-2$.

This includes the case of the special component on 3 rows. We have $r_{i}^{(1)} \geq 2$ by (17) and so $r_{i}+s_{i} \geq 2$. (Note that for $m \equiv 0(\bmod 4)$, each component in our construction from (11) has this property.)

Case 2. The number of columns non-constant on $C_{i}$ is $4 k-1$.

In this case, $r_{i}^{(1)} \geq 1$ by (17). We must carefully consider the maximum number of distinct non-constant columns on these $k$ rows using the argument of the Upper Bound Lemma 1.11. For $k \leq 3$, the non-constant columns all have either exactly one 1 or exactly one 0 or both when $k=2$. Then there are at most $2 k$ distinct such columns. The remaining $2 k-1$ columns must be non-canonical. Each such non-canonical column $\alpha$ is either $t$-varied for $t \geq 2$ and so $r_{i}^{(2)}(\alpha) \geq \frac{1}{2}$ or is 1 -varied and so $s_{i}(\alpha) \geq \frac{1}{k}$ from which we have $r_{i}^{(2)}(\alpha)+s_{i}(\alpha) \geq \frac{1}{k}$. Summing over the $2 k-1$ columns yields $r_{i}^{(2)}+s_{i} \geq 2$ since $(2 k-1) \frac{1}{k} \geq 1$ (for $k \geq 2$ ) and so $r_{i}^{(1)}+r_{i}^{(2)}+s_{i}=r_{i}+s_{i} \geq 2$. 
For $k \geq 4$, it is possible to have a non-constant column with at least two 1's and 0's on the rows of the component and such a column contributes at least $2(k-2)\left[\begin{array}{l}0 \\ 1\end{array}\right]$ configurations compared with $k-1$ for a column with just one 1 or just one 0 . For $k \geq 6$ there is at most one such column $(2(2(k-2))>3(k-1)$ for $k \geq 6)$ and for $k=5$ there are at most two such columns $(3(2(k-2))>4(k-1)$ for $k=5)$ and for $k=4$ there are at most three such columns $(4(2(k-2))>5(k-1)$ for $k=4)$. Thus there are at most $2 k+3$ distinct columns and these can be canonical with respect to $C_{i}$. There remain at least $2 k-4$ non-canonical columns. Now each of the at least $2 k-4$ non-canonical columns $\alpha$ have, as above, $r_{i}^{(2)}(\alpha)+s_{i}(\alpha) \geq \frac{1}{k}$ and so summing over the $2 k-4$ columns $\alpha$ we have $r_{i}^{(2)}+s_{i} \geq 1$ (since $(2 k-4) \frac{1}{k} \geq 1$ for $k \geq 4$ ) and then, using $r_{i}^{(1)} \geq 1$, we have $r_{i}^{(1)}+r_{i}^{(2)}+s_{i}=r_{i}+s_{i} \geq 2$.

Case 3. The number of columns non-constant on $C_{i}$ is $4 k$.

In this case we know by the argument of the Upper Bound Lemma 1.11 that the nonconstant columns will have either exactly one 1 or exactly one 0 (or both for $k=2$ ) on the rows of $C_{i}$. There are at most $2 k$ distinct such columns on the component (only 2 for $k=2$ ) and so at most $2 k$ canonical columns with respect to $C_{i}$ and so at least $2 k$ columns must be non-canonical. Each such column $\alpha$ has $r_{i}^{(2)}(\alpha)+s_{i}(\alpha) \geq \frac{1}{k}$ and so $r_{i}^{(2)}+s_{i} \geq 2$ by considering the $2 k$ columns $\alpha$. Thus $r_{i}+s_{i} \geq 2$. Note that the component of size $b$ in the construction (12) is covered by Case 3.

We have verified that $r_{i}+s_{i} \geq 2$ for any component and thus we have verified (23). This completes our proof.

We now consider $F_{2,4}$ and first obtain the analogues of Lemmas 3.1 and 3.2.

Lemma 3.10 For $F_{2,4}=\left[\begin{array}{cccccc}1 & 1 & 0 & 0 & 0 & 0 \\ 0 & 0 & 1 & 1 & 1 & 1\end{array}\right]$,

$$
\operatorname{forb}\left(m, F_{2,4}\right) \geq\left\lfloor\frac{10 m}{3}-\frac{4}{3}\right\rfloor \text {. }
$$

with equality for all $m \neq 2$.

Proof: We provide a construction for $A_{m}$, a simple matrix which avoids $F_{2,4}$. We may take $A_{1}=K_{1}, A_{3}=K_{3}$.

We can construct $A_{m}$ with no configuration $F_{2,4}$ using

$$
A_{m}=\left[\begin{array}{cccccc}
A_{m-k} & 1^{\prime} s & 1^{\prime} s & 0^{\prime} s & \vdots \\
& & & & 1 \\
& & & & 1 \\
0^{\prime} s & K_{k}^{1} & K_{k}^{k-1} & K_{k}^{1} & \vdots \\
& & & & 1
\end{array}\right] .
$$


We note that for $k=3$, if $A_{m-3}$ achieves the bound, then $A_{m}$ also achieves the bound (having 10 more columns). For $m=5$, we use (25) with $k=4$ to get a $5 \times 15$ matrix $A_{5}$ with $\left\lfloor\frac{10 \cdot 5}{3}-\frac{4}{3}\right\rfloor=15$. The rest of the cases can be constructed using (25) with $k=3$.

We do not expect uniqueness since for $m=4$ we may alternatively take $A_{4}$ to be

$$
A_{4}=\left[\begin{array}{llllll} 
& & 0 & 1 & 0 & 1 \\
K_{4}^{1} & K_{4}^{3} & 0 & 1 & 0 & 1 \\
& & 1 & 0 & 0 & 1 \\
& & 1 & 0 & 0 & 1
\end{array}\right]
$$

Let $A$ be a simple $m \times n$ matrix with no configuration $F_{2,4}$. We wish to show $n \leq \frac{10 m}{3}-\frac{4}{3}$ from which the bound follows. This is true for $m=1,2,3$ and $m=4$ by some easy case checking. We may assume it is impossible to delete $k$ rows and at most $\frac{10 k}{3}$ columns and still have a simple matrix $A_{m-k}$ by the Deletion Lemma 1.6.

We construct $D(A)$ as before.

Lemma 3.11 Our structure $D(A)$ can be assumed to have the following properties.

(i) Each pair of rows is connected by exactly one edge, directed or dotted.

(ii) The graph on the directed edges is transitive and contains no cycles.

(iii) All components of the graph on the dotted edges are cliques or possibly a three vertex component of two dotted edges.

Proof: With $p=2, q=4$ and $c=10 / 3$, we may appeal to Lemma 1.7 to deduce the properties (i),(ii). We now tackle the components of dotted edges. Assuming that there exists a component which is not a clique, there must be three rows $i, j, k$ with $i \longrightarrow j$, $k \cdots j, k \cdots i$ (hence $i, j, k$ are in the same component). Let us analyze this in detail. The possible entries for these rows are are

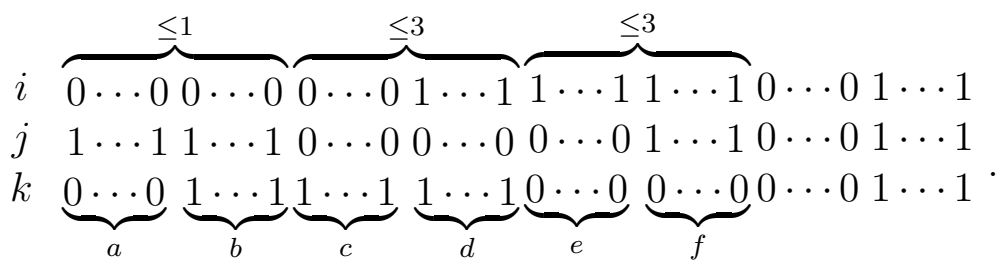

The total number of non-constant columns in these three rows is $\alpha=a+b+c+d+e+f$. Based on the three edges present we can see that $\alpha \leq 7$. If $\alpha \leq 6$ we are allowed to eliminate rows $j$ and $k$ and at most 6 columns to obtain a simple matrix but $\frac{6}{2} \leq \frac{10}{3}$ and so, by the Deletion Lemma 1.6 this does not occur. Hence $\alpha=7$ implying $a+b=1$, $c+d=3$ and $e+f=3$.

Removing a row must force removing 4 or more columns by the Deletion Lemma 1.6. If we wish to remove row $i$, then we can force the resulting $(m-1)$-rowed matrix to be simple by deleting the columns associated with $e$ and $b$ and ( $c$ or $d$ ) and ( $a$ or $f$ ). Thus, $a+b+e+\min \{c, d\} \geq 4$, yielding

$$
e+\min \{c, d\} \geq 3
$$


Removing row $j$ and the following combination of columns $d$ and $a$ and ( $e$ or $f$ ) and ( $b$ or $c$ ) results in a simple matrix. Thus, $a+b+d+\min \{e, f\} \geq 4$ yielding

$$
d+\min \{e, f\} \geq 3
$$

Removing row $k$ and the following combination of columns $d$ and $e$ and ( $a$ or $b$ ) and ( $c$ or $f$ ) results in a simple matrix. Thus, $f+c+\min \{a, b\}+\min \{d, e\} \geq 4$ and $\min \{a, b\}=0$ yielding

$$
f+c+\min \{d, e\} \geq 4 .
$$

We note $c+d=3$ implies $\min \{c, d\} \leq 1$ and similarly $e+f=3$ implies $\min \{e, f\} \leq 1$. Therefore, $e \geq 2, d \geq 2, c \leq 1$ and $f \leq 1$.

If $\min \{d, e\}=3 \geq 2$, then $d=e=3$ and $c=f=0$ which yields $f+c+\min \{d, e\}=$ $3 \ngtr 4$, a contradiction. On the other hand, if $\min \{d, e\}=2$ then $f+c \geq 2$ which forces $c=f=1$ and $d=e=2$.

We now consider the components in the graph formed by the dotted edges. We first note that any components of size $k=1,2,3$ must be a clique of dotted edges or our special component of two dotted edges on three vertices whose columns were analyzed above.

Suppose we have a component, not a clique, with 4 or more vertices. There must be at least two vertices at distance two. Assume the two vertices $i, j$ have a shortest path $i, k, j$ of dotted edges joining them. There must be a directed edge connecting $i$ and $j$. Since the two are interchangeable, the following configuration takes place:

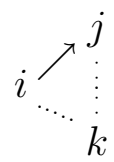

Let $l$ be any other vertex of the component and on the four rows we have the situation below. We will arrive at a contradiction.

$$
\begin{array}{cccccccc}
i & 0 & 0 & 11 & 11 & 1 & 0 \cdots 0 & 1 \cdots 1 \\
j & 1 & 0 & 00 & 00 & 1 & 0 \cdots 0 & 1 \cdots 1 \\
k & 0 \text { or } 1 & 1 & 11 & 00 & 0 & 0 \cdots 0 & 1 \cdots 1 \\
l & L & C & D & E & F & G & H
\end{array}
$$

where $L, C, F$ are $1 \times 1$ matrices, $D, E$ are $1 \times 2$ matrices and $G, H$ are 1 -rowed matrices. We note that if $\# 1^{\prime} s(G)+\# 0^{\prime} s(H) \leq 3$ then we could delete the 3 rows $i, j, k$ and the up to 10 columns non-constant on rows $i, j, k, l$ to obtain a simple matrix and so violate the Deletion Lemma 1.6. We conclude

$$
\# 1^{\prime} s(G)+\# 0^{\prime} s(H) \geq 4
$$

We show that the following four cases do not occur.

Case 1. $i \cdots \cdot l, j \cdots \cdot l$ are both present.

Using our previous result for the triple $(i, j, l)$, we deduce $\# 1^{\prime} s(C G)=1$ and $\# 0^{\prime} s(F H)=1$. But then $\# 1^{\prime} s(G)+\# 0^{\prime} s(H) \leq 2$ contradicting (27). Thus Case 1 does not occur. 
Case 2. $i \cdots \cdot l, l \rightarrow j, l \rightarrow k$ are all present.

We note that in $(26)$ with triple $(i, j, k)$ replaced by the triple $(l, k, i)$, the number of columns with a 0 in row $i$ and a 1 in row $k$ is at most 2. Also the number of columns with a 1 in row $i$, a 1 in row $j$ and a 0 in row $k$ is 1 . We deduce $\# 1^{\prime} s(L G) \leq 2$ and $\# 0^{\prime} s(D H)=1$. But then $\# 1^{\prime} s(G)+\# 0^{\prime} s(H) \leq 3$ which contradicts $(27)$. Thus Case 2 does not occur.

Case 3. $l \rightarrow j, l \cdots k$ are both present.

Considering the triple $(l, j, k)$, we discover that $\# 1^{\prime} s(E G)=2$ and $\# 0^{\prime} s(L H) \leq 1$. But then we would have $\# 1^{\prime} s(G)+\# 0^{\prime} s(H) \leq 3$ which contradicts (27). Thus Case 3 does not occur.

Case 4. $i \rightarrow l, l \ldots k$ cannot both occur.

Considering the triple $(i, l, k)$, we discover that $\# 1^{\prime} s(L G) \leq 1$ and $\# 0^{\prime} s(D H)=2$. Thus, \#1's $(G)+\# 0^{\prime} s(H) \leq 3$ which contradicts (27). Thus Case 4 does not occur.

We now consider the components in the graph formed by the dotted edges. We wish to show that any components of size at least 4 must be a complete clique of dotted edges. Suppose our component above is not a clique with a triple $(i, j, k)$ with structure $i \rightarrow j$, $i \cdots k, j \cdots k$ and where $l$ is any other vertex of the same component. Following a similar argument used in Lemma 3.2, we show, using the four cases, that either $i \rightarrow l, j \rightarrow l$ and $k \rightarrow l$ or $l \rightarrow i, l \rightarrow j$ and $l \rightarrow k$. This cannot be true for all choices of $l$ and based on this contradiction the component has to be a clique.

If $l \rightarrow i$, transitivity will force $l \rightarrow j$. Also by transitivity, $k \rightarrow l$ cannot exist since it would force $k \rightarrow i$ which would violate property (i). Case 3 eliminates $l \cdots k$ and thus we must have $l \rightarrow k$ in which case all edges from $l$ are directed to $i, j, k$.

If $l \cdots i$ then $j \rightarrow l$ may not happen based on transitivity. Case 1 eliminates having $l \cdots \cdots j$. The remaining possibility is $l \rightarrow j$ which then prevents having $k \rightarrow l$ from happening. Case 2 now eliminates $l \rightarrow k$ and Case 3 eliminates $l \cdots k$. So this cannot occur.

If $i \rightarrow l$ then transitivity forces $l \rightarrow k$ not to occur. Case 4 eliminates the possibility of having $l \cdots k$. Now, if $k \rightarrow l$, then transitivity forbids $l \rightarrow j$. Case 2 already covers $l \cdots j$ and thus we have $j \rightarrow l$. All edges are directed into $l$.

As promised, we have shown for any other vertex $l$ of the same component that there are no dotted edges joining $l$ to $i, j, k$ for any vertex $l$ in the same component. This contradicts that $i, j, k$ is in a component with any other vertices. We have established property (iii).

By the Ordering Lemma 1.8 there is a linear ordering of the components $C_{1}, C_{2}, C_{3}, \ldots$ so that for $i, j$ with $i<j$ we have for all $x \in C_{i}$ and $y \in C_{j}$ there is the directed edge $x \rightarrow y$. Reorder the rows of $A$ to respect this linear order of components. In obtaining the bound for $F_{2,4}$ we follow the proof outline of Theorem 3.9 but with some refinements in the consideration of $r+s$. We believe the greater number of extremal constructions is responsible for the more delicate proof.

We add an additional idea that was not necessary to prove Theorem 3.9.

Definition 3.12 If there is a non-canonical 1-varied column which is non-constant on $C_{i}$ and the column has either a 0 on $C_{j}$ (and hence all 0 's if $j \neq i$ ) and all 1 's on $C_{j+1}$ or 
all 0's on $C_{j}$ and a 1 on $C_{j+1}$ (and hence all 1 's if $j+1 \neq i$ ) then we say that component $C_{i}$ uses $\left(C_{j}, C_{j+1}\right)$.

Claim 3.13 If component $C_{i}$ uses $\left(C_{j}, C_{j+1}\right)$ then no other component uses $\left(C_{j}, C_{j+1}\right)$.

Proof: This is readily seen for $j, j+1 \neq i$. If $j=i$ or $j+1=i$ then one verifes that no other component uses $\left(C_{j}, C_{j+1}\right)$. In particular if $i=j$ and $C_{i}$ uses $\left(C_{j}, C_{j+1}\right)$ then there is a 1-varied column $\alpha$ non-constant on $C_{i}=C_{j}$ and a row $a$ of $C_{i}=C_{j}$ with $\alpha$ having a 0 on row $a$ and 1's on rows of $C_{j+1}$. If $C_{j+1}$ uses $\left(C_{j}, C_{j+1}\right)$ then there is a 1-varied column $\beta$ non-constant on $C_{j+1}$ and a row $b$ of $C_{j+1}$ with $\beta$ having a 1 on row $b$ and all 0's on $C_{j}=C_{i}$. This creates the submatrix $\left[\begin{array}{ll}0 & 0 \\ 1 & 1\end{array}\right]$ on rows $a, b$. This contradiction proves the claim.

We will use the fact that at most $m^{\prime}-1$ pairs $\left(C_{j}, C_{j+1}\right)$ can be used. It remains true that up to $\left|C_{i}\right|$ non-canonical 1-varied columns non-constant on $C_{i}$ can each use the same $\left(C_{j}, C_{j+1}\right)$ (where $j=i$ or $j+1=i$ ) such as outlined by the following two possibilities each of $\left|C_{i}\right|$ columns where we only give the two components of interest (you can imagine the remaining entries filled with 1's above and 0's below):

$$
C_{i-1}\left[\begin{array}{c}
1^{\prime} s \\
0^{\prime} s \\
K_{\left|C_{i}\right|}^{1} \\
0^{\prime} s
\end{array}\right] \text { or } C_{i+1}\left[\begin{array}{c}
1_{i}^{\prime} s \\
K_{\left|C_{i}\right|}^{\left|C_{i}\right|-1} \\
1^{\prime} s \\
0^{\prime} s
\end{array}\right]
$$

Theorem 3.14 For $F_{2,4}=\left[\begin{array}{cccccc}1 & 1 & 0 & 0 & 0 & 0 \\ 0 & 0 & 1 & 1 & 1 & 1\end{array}\right]$

$$
\operatorname{forb}\left(m, F_{2,4}\right) \leq\left\lfloor\frac{10 m}{3}-\frac{4}{3}\right\rfloor
$$

with equality for all $m \neq 2$.

Proof: In view of Lemma 3.10 we need only establish the upper bound and in view of Lemma 3.11 we may assume all components are cliques. We follow the proof of Theorem 3.9 and use the Ordering Lemma 1.8 to order the components.

Using Claims 3.5 and 3.7 together, $A$ has a maximum of $3\left(m-c_{1}\right)+2 m^{\prime}-(r+s)$ columns. Using (14) we note

$$
3\left(m-c_{1}\right)+2 m^{\prime}=2 c_{1}+\sum_{k \geq 2}(3 k+2) c_{k}=2 c_{1}+\sum_{k \geq 2} \frac{10 k}{3} c_{k}+\sum_{k \geq 2}\left(2-\frac{k}{3}\right) c_{k} .
$$

We will show below that

$$
r+s \geq \sum_{k \geq 2}\left(2-\frac{k}{3}\right) c_{k}+\delta
$$


with $\delta \geq 0$ where, in addition if $c_{1}=0$, we have

$$
\delta> \begin{cases}1 & \text { if } m \equiv 0(\bmod 3) \\ \frac{1}{3} & \text { if } m \equiv 1(\bmod 3) \\ \frac{2}{3} & \text { if } m \equiv 2(\bmod 3)\end{cases}
$$

This would mean

$$
n \leq 2 c_{1}+\sum_{k \geq 2} \frac{10 k}{3} c_{k}+\sum_{k \geq 2}\left(2-\frac{k}{3}\right) c_{k}-\sum_{k \geq 2}\left(2-\frac{k}{3}\right) c_{k}-\delta \leq \frac{10 m}{3}-\delta-\frac{4}{3} c_{1}
$$

Thus checking cases yields

$$
n \leq\left\lfloor\frac{10 m}{3}-\frac{4}{3}\right\rfloor
$$

if either $c_{1} \geq 1$ or $(31)$ is true.

Following the proof of Theorem 3.9, our argument considers five cases and establishes inequalities on $r_{i}+s_{i}$ in each case. For every component $C_{i}$ with $\left|C_{i}\right| \geq 2$ we show that $r_{i}+s_{i} \geq 2-\left(\left|C_{i}\right| / 3\right)$. This is sufficient to prove (30) if either $c_{1} \geq 1$ or we only had to show that $\delta=0$. If $c_{1}=0$, we need (31) to establish (28). We can often find a component $C_{i}$ with $r_{i}+s_{i}>2-\left|C_{i}\right| / 3+\epsilon$ where $\epsilon$ corresponds to $\delta$ when we replace $m$ by $\left|C_{i}\right|$ which becomes helpful for our proof. We also obtain stronger inequalities in the case of a component that uses no pair $\left(C_{k}, C_{k+1}\right)$. We note that there are only $m^{\prime}-1$ pairs $\left(C_{j}, C_{j+1}\right)$ which can be used and for components that use no pair $\left(C_{j}, C_{j+1}\right)$ we are able to get stronger inequalities and results, enabling us to prove (30). Our most difficult cases arise with all components of size 3 .

Note that in the case that $C_{i}$ is a clique and has $3\left|C_{i}\right|$ columns non-constant on $C_{i}$, then by the proof of the Upper Bound Lemma 1.11, we deduce that all such columns have either exactly one 0 on $C_{i}$ or exactly one 1 on $C_{i}$. Of course for $\left|C_{i}\right|=2$ or 3 , this is trivially true.

Case 1. $\left|C_{i}\right|=2$.

The maximum number of distinct non-constant columns on this component is 2 . If at most 3 columns are non-constant then $r_{i}^{(1)} \geq 3$. If there are 4 non-constant columns, then $r_{i}^{(1)}=2$ and also two columns are non-canonical. A non-canonical column $\alpha$ is either $t$-varied for $t \geq 2$ and hence $r_{i}^{(2)}(\alpha) \geq \frac{1}{2}$ and $s_{i}(\alpha)>0$ or $\alpha$ is 1 -varied and $s_{i}(\alpha) \geq \frac{1}{2}$ and in addition some pair $\left(C_{j}, C_{j+1}\right)$ is used. Summing over the two non-canonical columns and adding in $r_{i}^{(1)}$, we obtain $r_{i}+s_{i} \geq 3$. If there are 5 non-constant columns, then $r_{i}^{(1)}=1$ and also three columns are non-canonical. As above, each such column $\alpha$ has either $r_{i}^{(2)}(\alpha) \geq \frac{1}{2}$ and $s_{i}(\alpha)>0$ or $s_{i}(\alpha) \geq \frac{1}{2}$ and some pair $\left(C_{j}, C_{j+1}\right)$ is used. Summing over the three non-canonical columns and adding in $r_{i}^{(1)}$, we obtain $r_{i}+s_{i} \geq 5 / 2$.

If there are 6 non-constant columns, then four columns must be non-canonical. As above, each such column $\alpha$ has either $r_{i}^{(2)}(\alpha) \geq \frac{1}{2}$ and $s_{i}(\alpha)>0$ or $s_{i}(\alpha) \geq \frac{1}{2}$ and some pair $\left(C_{j}, C_{j+1}\right)$ is used. Summing over the four non-canonical columns we obtain $r_{i}+s_{i} \geq 2$ 
and we will only have equality if $s_{i}=2$ and $C_{i}$ will have used two pairs $\left(C_{j}, C_{j+1}\right)$ and $\left(C_{k}, C_{k+1}\right)$.

In summary, we have $r_{i}+s_{i} \geq 2-\left|C_{i}\right| / 3+2 / 3=2$ with $r_{i}+s_{i}>2$ if no pair $\left(C_{j}, C_{j+1}\right)$ is used.

Case 2. $\left|C_{i}\right|=3$ and $C_{i}$ is a special component.

Such a component has 7 columns which are non-constant on $C_{i}$, yielding $r_{i}^{(1)}=2$, and also two non-canonical columns in (26) since $d=e=2$. Each non-canonical column $\alpha$ has either $r_{i}^{(2)}(\alpha) \geq \frac{1}{2}$ and $s_{i}(\alpha)>0$ or $s_{i}(\alpha) \geq \frac{1}{3}$ and some pair $\left(C_{j}, C_{j+1}\right)$ is used. Summing over the two non-canonical columns and adding in $r_{i}^{(1)}$, we obtain $r_{i}+s_{i} \geq \frac{8}{3}>$ $2-\left|C_{i}\right| / 3+1=2$.

Case 3. $\left|C_{i}\right|=3$ and $C_{i}$ is a clique.

We note that there are 8 possible columns on 3 rows of which exactly 6 columns are non-constant. If there are at most 6 columns of $A$ canonical with respect to $C_{i}$, then $r_{i}^{(1)} \geq 3$. If there are 7 columns non-constant on $C_{i}$ (yielding $r_{i}^{(1)}=2$ ), then there must be one non-canonical column $\alpha$ and it has either $r_{i}^{(2)}(\alpha) \geq \frac{1}{2}$ and $s_{i}(\alpha)>0$ or $s_{i}(\alpha) \geq \frac{1}{3}$ and some pair $\left(C_{j}, C_{j+1}\right)$ is used. Now $r_{i}^{(2)}=r_{i}^{(2)}(\alpha)$ and $s_{i}=s_{i}(\alpha)$. Adding in $r_{i}^{(1)}$, we obtain $r_{i}+s_{i} \geq \frac{7}{3}>2-\left|C_{i}\right| / 3+1=2$. If there are 8 columns non-constant on $C_{i}$ (yielding $r_{i}^{(1)}=1$ ), then there must be two non-canonical columns. Each such column $\alpha$ has either $r_{i}^{(2)}(\alpha) \geq \frac{1}{2}$ and $s_{i}(\alpha)>0$ or $s_{i}(\alpha) \geq \frac{1}{3}$ and some pair $\left(C_{j}, C_{j+1}\right)$ is used. Summing over the two non-canonical columns and adding in $r_{i}^{(1)}$, we obtain $r_{i}+s_{i} \geq \frac{5}{3}$. Moreover $r_{i}+s_{i}>2$ unless we have used some pair $\left(C_{j}, C_{j+1}\right)$.

If there are 9 columns non-constant on $C_{i}$, then there must be 3 non-canonical columns. Each such column $\alpha$ has either $r_{i}^{(2)}(\alpha) \geq \frac{1}{2}$ and $s_{i}(\alpha)>0$ or $s_{i}(\alpha) \geq \frac{1}{3}$ and some pair $\left(C_{j}, C_{j+1}\right)$ is used. Summing over the three non-canonical columns we obtain $r_{i}+s_{i} \geq 1$. Several cases have $r_{i}+s_{i} \leq 2$ and we see that $r_{i}+s_{i}>3 / 2$ unless $C_{i}$ has used some pair $\left(C_{j}, C_{j+1}\right)$.

Summarizing we have $r_{i}+s_{i} \geq 2-\left|C_{i}\right| / 3=1$ in all cases and moreover the only cases with $r_{i}+s_{i} \leq 2-\left|C_{i}\right| / 3+1=2$ are the following four cases: either $r_{i}=3 / 2, s_{i}>0$ or $r_{i}=1, s_{i}>1 / 3$, or $r_{i}=1 / 2, s_{i}>2 / 3$, or $r_{i}=0, s_{i}=1$. Note that the only case not using any pair $\left(C_{j}, C_{j+1}\right)$ is to have $r_{i}=3 / 2, s_{i}>0$. Also note that the cases with $r_{i}+s_{i}<2-\left|C_{i}\right| / 3+1=2$ and having $r_{i}>0$ must occur with $t$-varied columns (with $t \geq 2$ ) which are non-constant on $C_{i}$ and so non-constant on some other components $C_{j}$ which forces $r_{j}>0$.

Case 4. $\left|C_{i}\right|=4,5,6$.

If there are at most $3\left|C_{i}\right|-2$ columns of $A$ non-constant on $C_{i}$, then $r_{i}^{(1)} \geq 2$ so $r_{i}+s_{i} \geq 2$.

If there are $3\left|C_{i}\right|-1$ columns of $A$ non-constant on $C_{i}$ then $r_{i}^{(1)}=1$ and if there is a non-canonical column this forces $r_{i}+s_{i}>1$. To show that there is a non-canonical column, we use Theorem 2.1 and compute forb $\left(4, F_{0,4}\right)=12$, forb $\left(5, F_{0,4}\right)=14$, forb $\left(6, F_{0,4}\right)=17$. If we had $3\left|C_{i}\right|-1$ distinct non-constant columns on $\left|C_{i}\right|$ rows, then we could add the two constant columns to get a simple $\left|C_{i}\right| \times\left(3\left|C_{i}\right|+1\right)$ matrix with no $F_{0,4}$, a contradiction 
for these values.

If there are $3\left|C_{i}\right|$ columns of $A$ non-constant on $C_{i}$, then the columns either have exactly one 1 or exactly one 0 and so there must be $\left|C_{i}\right|$ non-canonical columns and each such column $\alpha$ either is $t$-varied for $t \geq 2$ and so has $r_{i}^{(2)}(\alpha) \geq 1 / 2$ and $s_{i}(\alpha)$ or $\alpha$ is 1-varied $s_{i}(\alpha) \leq 1 /\left|C_{i}\right|$ and some pair $\left(C_{j}, C_{j+1}\right)$ is used by $C_{i}$.

Summarizing, in all cases, $r_{i}+s_{i} \geq 1>2-\left|C_{i}\right| / 3$. For $\left|C_{i}\right|=4$, this yields $r_{i}+s_{i} \geq$ $2-\left|C_{i}\right| / 3+1 / 3=1$. For $\left|C_{i}\right|=5$, this yields $r_{i}+s_{i} \geq 2-\left|C_{i}\right| / 3+2 / 3=1$. For $\left|C_{i}\right|=6$, this yields $r_{i}+s_{i} \geq 2-\left|C_{i}\right| / 3+1=1$. Moreover in each of these three situations, we will have strict inequality if no pair $\left(C_{j}, C_{j+1}\right)$ is used.

Case 5. $\left|C_{i}\right| \geq 7$.

We note that $2-\left|C_{i}\right| / 3+1<0$. Moreover it easy to show that $r_{i}+s_{i} \geq 1$ since either there are at most $3\left|C_{i}\right|-1$ columns of $A$ non-constant on $C_{i}$ and so $r_{i}^{(1)} \geq 1$ or there are $3\left|C_{i}\right|$ such columns and we can use the argument of Case 4. Summarizing, $r_{i}+s_{i}>2-\left|C_{i}\right|+1$.

We have verified for any component $C_{i}$ of size at least 2 that $r_{i}+s_{i} \geq 2-\left(\left|C_{i}\right| / 3\right)$ with strict inequality if no pair is used. This establishes the bulk of (30) if we are only required to show $\delta=0$. We do not have to verify (31) for $c_{1} \geq 1$. If there is a component $C_{i}$ with:

$$
r_{i}+s_{i}>2-\left|C_{i}\right|- \begin{cases}1 & \text { if }\left|C_{i}\right| \equiv 0(\bmod 3) \\ \frac{1}{3} & \text { if }\left|C_{i}\right| \equiv 1(\bmod 3) \\ \frac{2}{3} & \text { if }\left|C_{i}\right| \equiv 2(\bmod 3)\end{cases}
$$

then we can use this to establish (31) in some cases. Note that in general $\left|C_{i}\right| \not \equiv m(\bmod 3)$ so our arguments consider the possibilities for $m$ modulo 3 .

Now (34) is true for $\left|C_{i}\right| \geq 7$ or $C_{i}$ is a special component on 3 rows. Also, if $C_{i}$ uses no pair, then (34) is true for $\left|C_{i}\right|=2,4,5$, or 6 . If $\left|C_{i}\right|=2,4,5$, or 6 and $C_{i}$ uses some pair $\left(C_{j}, C_{j+1}\right)$, then we get $(34)$ with $>$ replaced by the weaker inequality $\geq$ but this is still useful.

For $m \equiv 1(\bmod 3)$ there is either a component $C_{i}$ with $\left|C_{i}\right| \equiv 1(\bmod 3)$ or two components $C_{i}, C_{j}$ with $\left|C_{i}\right|,\left|C_{j}\right| \equiv 2(\bmod 3)$. This, and (34) establishes $(31)$ without the strict inequality but then we use the fact that some component uses no pair $\left(C_{j}, C_{j+1}\right)$ and this gives us (31) with strict inequality.

For $m \equiv 2(\bmod 3)$ there is either a component $C_{i}$ with $\left|C_{i}\right| \equiv 2(\bmod 3)$ or two components $C_{i}, C_{j}$ with $\left|C_{i}\right|,\left|C_{j}\right| \equiv 1(\bmod 3)$. This, and (34) establishes $(31)$ without the strict inequality but then we use the fact that some component uses no pair $\left(C_{j}, C_{j+1}\right)$ and this gives us (31) with strict inequality.

The case $m \equiv 0(\bmod 3)$ is trickier. We would be done if there is a component $C_{i}$ with $\left|C_{i}\right| \equiv 1(\bmod 3)$ and a component $C_{j}$ with $\left|C_{j}\right| \equiv 2(\bmod 3)$, obtaining $(31)$ from our case analysis without the strict inequality but again we use the fact that some component uses no pair $\left(C_{j}, C_{j+1}\right)$ to obtain (31) with strict inequality. Similar arguments work for three components of size $\equiv 1(\bmod 3)$ or three components of size $\equiv 2(\bmod 3)$ or indeed a component of size 6 or greater or a special component of size 3 . 
We may now assume all components are cliques of size 3 and each component $C$ has 9 varied columns non-constant on $C$. Since some component $C_{i}$ uses no pair $\left(C_{j}, C_{j+1}\right)$, we deduce for that component $r_{i}=3 / 2, s_{i}>0$ (or we are done by our summary of Case 3 ). Thus there must be other components $C_{j}$ with $r_{j}>0$ since we obtained $r_{i}=3 / 2$ from three $t$-varied columns (with $t \geq 2$ ) each non-constant on $C_{i}$. If we have another component $C_{j}$ with $r_{j}=3 / 2$ and $s_{j}>0$, then $r_{i}+s_{i}+r_{j}+s_{j}>3$ which establishes (31) using that we already have $r_{l}+s_{l} \geq 2-\left|C_{l}\right| / 3$ for all $l$. Similarly if we have two components $C_{j}, C_{l}$ with $r_{j}=1 / 2, s_{j}>2 / 3$ and $r_{l}=1, s_{l}>1 / 3$, then we have $r_{i}+r_{j}+r_{l}=3, s_{i}+s_{j}+s_{l}>1$ yielding $\sum_{t \in\{i, j, l\}}\left(r_{t}+s_{t}\right)>1+\sum_{t \in\{i, j, l\}}\left(2-\left|C_{t}\right| / 3\right)=4$ and hence establishing (31). (This is not a hypothetical case; one can construct a $9 \times 28$ simple matrix with no $F_{2,4}$ with three components of size 3 , the first having $r_{i}=1 / 2, s_{i}>2 / 3$, the middle component with $r_{i}=3 / 2, s_{i}>0$ and the last component with $r_{l}=1, s_{l}>1 / 3$ ). Similar arguments establish the remaining cases such as 3 components $C_{j}, C_{k}, C_{l}$ with $r_{j}=r_{k}=r_{l}=1 / 2$ and $s_{j}>2 / 3, s_{k}>2 / 3$, and $s_{l}>2 / 3$. We have $r_{i}+r_{j}+r_{k}+r_{l}=3, s_{i}+s_{j}+s_{k}+s_{l}>2$ yielding $\sum_{t \in\{i, j, k, l\}}\left(r_{t}+s_{t}\right)>1+\sum_{t \in\{i, j, k, l\}}\left(2-\left|C_{t}\right| / 3\right)=5$ and hence establishing (31).

Thus (30) is confirmed and our proof is complete.

Examining the cases of equality in the proof leads to a variety of constructions achieving the bound. Note that a component $C_{i}$ of size 3 with $s_{i}=1$ fits easily into such a construction.

\section{General Construction}

We now consider the general $F_{p q}$ with $l=q-p$. The following construction may be close to best possible for $l \geq 8$ and shows that forb $\left(m, F_{p q}\right)$ is $\Omega\left(\left(\frac{p+q}{2}+O(1)\right) m\right)$. Choose $k=\lfloor l / 2\rfloor+1$. We construct $D_{k}$ as a $k \times k(k+1)$ matrix:

$$
D_{k}=\left[\begin{array}{llll}
K_{k}^{1} & K_{k}^{2} & K_{k}^{k-2} & K_{k}^{k-1}
\end{array}\right]
$$

This has $k(k+1)$ columns if $k \geq 5$ and hence if $l \geq 8$. Note that a pair of rows of $K_{k}^{1}$ has one copy of $F_{1,1}$ and $K_{k}^{2}$ has $F_{k-2, k-2}$. Thus $D_{k}$ has $F_{2(k-1), 2(k-1)}=F_{2\left\lfloor\frac{l}{2}\right\rfloor, 2\left\lfloor\frac{l}{2}\right\rfloor}$ but no configuration $F_{2\left\lfloor\frac{l}{2}\right\rfloor, 2\left\lfloor\frac{l}{2}\right\rfloor+1}$. Following the construction idea in Theorem 3.6 in [2] for matrices with no submatrix $\left[\begin{array}{lllll}0 & 0 & 0 & \ldots & 0 \\ 1 & 1 & 1 & \cdots & 0\end{array}\right]$ (row and column order matter when discussing a submatrix), we form the following matrices where individual $1 \times 1$ entries have been replaced by appropriate $k \times k$ matrices in an interesting way. Assume $m \equiv 0(\bmod k)$. Each $B_{i}$ will be of size $m \times(m-k)$. 


$$
B_{i}=\left[\begin{array}{ccccccccc}
K_{k}^{k-1} & & & & & & & \\
1^{\prime} s & K_{k}^{k-1} & & & & & 1^{\prime} s & \\
1^{\prime} s & 1^{\prime} s & \ddots & & & & & \\
\vdots & 1^{\prime} s & \ddots & K_{k}^{k-1} & & & & \\
\vdots & \vdots & \ddots & 1^{\prime} s & 0^{\prime} s & 0^{\prime} s & \cdots & 0^{\prime} s \\
1^{\prime} s & \vdots & & 1^{\prime} s & K_{k}^{1} & 0^{\prime} s & \cdots & 0^{\prime} s \\
& 1^{\prime} s & & \vdots & 0^{\prime} s & K_{k}^{1} & \cdots & 0^{\prime} s \\
& & \ddots & \vdots & \vdots & \vdots & \ddots & \vdots \\
& 0^{\prime} s & & 1^{\prime} s & 0^{\prime} s & 0^{\prime} s & \cdots & K_{k}^{1}
\end{array}\right] .
$$

Following the arguments in [2], we can check that each $B_{i}$ has, for any pair of rows $i, j$ with $i<j$, at most one column with ${ }_{j}^{i}\left[\begin{array}{l}0 \\ 1\end{array}\right]$. Thus $\left[B_{1} B_{2} \cdots B_{p-1}\right]$ has no submatrix of $p$ copies of ${ }_{j}^{i}\left[\begin{array}{l}0 \\ 1\end{array}\right]$.

We now form a simple $m$-rowed matrix $A$ as below with no $F_{p q}$. of size $m \times(\lfloor l / 2\rfloor+$ $2+(p-1)+1 /(\lfloor l / 2\rfloor+1)) m-(p-1)(\lfloor l / 2\rfloor+1)+1$. We use the notation that $\mathbf{1}$ is a column of $k 1$ 's and $\mathbf{0}$ is a column of $k 0$ 's:

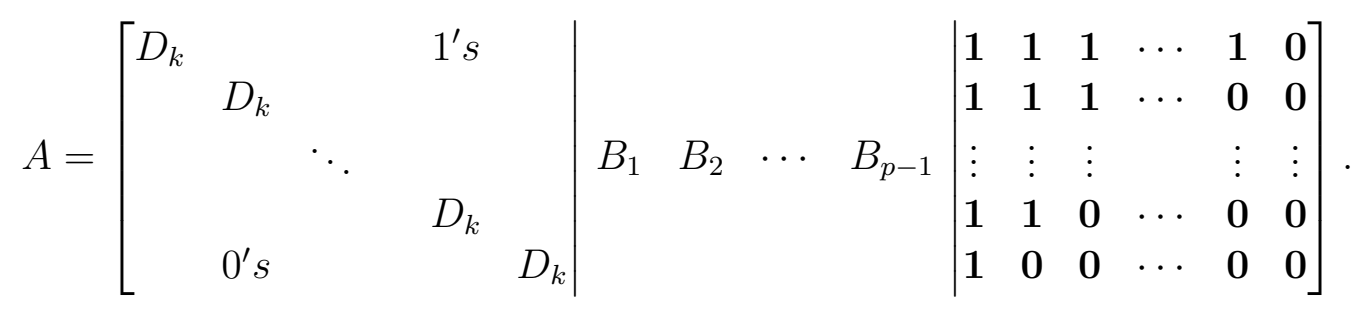

The number of columns containing the $D_{k}$ 's is $(m / k)$ times $k(k+1)$ which is $(\lfloor l / 2\rfloor+$ $2) m$. The number of columns from the $B_{i}$ 's is $(p-1)(m-k)$ which is $(p-1) m-(p-$ $1)(\lfloor l / 2\rfloor+1)$. There are $1+m / k$ final columns which is $\frac{1}{([l / 2\rfloor+1)} m+1$. Our construction is seen to have $\Theta((p+q) / 2+O(1)) m)=$ columns. We are using $O(1)$ for fixed $p, q$ as $m \rightarrow \infty$.

Theorem 4.1 Assume $1 \leq p<q$ are given with $q-p=l \geq 8$. Then $\operatorname{forb}\left(m, F_{p q}\right) \geq(\lfloor l / 2\rfloor+1+p+1 /(\lfloor l / 2\rfloor+1)) m-(p-1)(\lfloor l / 2\rfloor+1)+1$.

Proof: We have indicated how the number of columns in $A$ is computed. We need to show that $A$ has no $F_{p q}$. Consider two rows $i, j$ of $A$ with $i<j$. If $i, j$ are in the same block of $k=\left\lfloor\frac{l}{2}\right\rfloor+1$ rows, then inside the first $\left(\left\lfloor\frac{l}{2}\right\rfloor+1\right) m$ columns of $A$, one only looks inside a copy of $D_{k}$ which has $F_{2(k-1), 2(k-1)}=F_{2\left\lfloor\frac{l}{2}\right\rfloor, 2\left\lfloor\frac{l}{2}\right\rfloor}$ but no configuration $F_{2\left\lfloor\frac{l}{2}\right\rfloor, 2\left\lfloor\frac{l}{2}\right\rfloor+1}$. But in $\left[B_{1} B_{2} \cdots B_{p-1}\right]$, there is no submatrix of $p$ copies of $\left[\begin{array}{l}0 \\ 1\end{array}\right]$ and so rows $i, j$ of $A$ do not have the configuration $F_{0, p+2\lfloor l / 2\rfloor}$ and so $A$ has no $F_{p q}$. 
If $i, j$ are from different blocks then there are many copies of the submatrix ${ }_{j}^{i}\left[\begin{array}{l}1 \\ 0\end{array}\right]$ but the only copies of ${ }_{j}^{i}\left[\begin{array}{l}0 \\ 1\end{array}\right]$ are in $\left[B_{1} B_{2} \cdots B_{p-1}\right]$ which does not have $p$ copies of the submatrix ${ }_{j}^{i}\left[\begin{array}{l}0 \\ 1\end{array}\right]$. Thus there is no configuration $F_{p q}$.

The proofs in this paper provided motivation for the construction. Note that the 'components' are each a consecutive set of $k$ rows and they are arranged with transitivity holding as in Lemma 1.8 (we have no proof that transitivity holds in general).

One can also use the construction with small values of $l$ by making alternative choices for $D_{k}$. For $l=0$, the choice of $k=1$ and $D_{1}$ to have no columns yields a known optimal solution [3]. For $l=1$, a natural choice would be $k=2$ and $D_{2}=K_{2}^{1}$. For $l=2$, we might take $k=3$ and $D_{3}=\left[K_{3}^{1} K_{3}^{2}\right]$. For $l=3$, we might take $k=4$ and $D_{4}=\left[K_{4}^{1} M_{4} K_{4}^{3}\right]$. One can continue to propose the appropriate choices for larger $l$ but perhaps this should wait until some optimal bounds are proven.

\section{Acknowledgements}

Many thanks to an extremely careful and patient referee for encouraging substantial reorganizations of the paper which hopefully aids readability. Any remaining problems are mine.

\section{References}

[1] R.P. Anstee, J.R. Griggs, A. Sali, Small Forbidden Configurations, Graphs and Combinatorics 13(1997),97-118.

[2] R.P. Anstee, Z. Füredi, Forbidden Submatrices, Discrete Math. 62(1986),225-243.

[3] R.P. Anstee, R. Ferguson, A. Sali, Small Forbidden Configurations II, Electronic J. Combin. 8(2001), R4 (25pp).

[4] Z. Füredi, P. Hajnal, Davenport-Schinzel Theory, J. Combin. Th. Ser. A. 111(2005),266-288.

[5] M. Karpinski, T. Werther, VC-dimension and uniform learnability of sparse polynomials and rational functions, SIAM J. Comput. 22(1993), 1276-1285.

[6] Jiři Matoušek, Bounded VC-dimension implies a fractional Helly Theorem, Discrete Comput. Geom. 31(2004), 251-255.

[7] G. Tardos, On (0,1)-matrices and small excluded Submatrices, Discrete Math. 62(1986),225-243. 